\title{
A LOGIC OF HYPOTHETICAL CONJUNCTION
}

\author{
MATTHEW COLLINSON
}

\begin{abstract}
A binary connective that can be read as a matching conjunction for conditional connectives found in many conditional logics is considered. The most natural way to read this connective is often as a conjunction and yet, hypothetically, considered to hold of a state-of-affairs that could obtain under the hypothesis. The connective can be given an intensional semantics extending a standard semantics of conditional logic that uses propositionally indexed families of binary relations on possible worlds. This semantics is determined by an adjoint relationship between the operations supporting the semantics of the conditional and the new conjunction. The semantics of the hypothetical conjunction connective subsumes the semantics, supported by a ternary relation semantics, of the fusion connective that arises in connection with substructural and relevant logics, and therefore subsumes a number of other forms of conjunction. A number of applications of the hypothetical conjunction connective are discussed, including to generalized forms of resource reasoning of use in computer science applications.
\end{abstract}

\section{INTRODUCTION}

A number of logics feature conditional formulae of the form $\phi \rightarrow \psi$ in which the antecedent (hypothesis) $\phi$ is naturally read as carrying information about situations in which the consequent $\psi$ is to obtain. Methods from intensional logic, specifically possible worlds semantics, have given a great deal of insight into such conditionals, and in recent years have read to diverse applications.

A simple version of such a semantics uses frames consisting of a set of worlds, $W$, and a binary relation $R_{\phi}$ on $W$ for each propositional formula $\phi$. The condition for $\phi \rightarrow \psi$ to hold at world $w$ is then that $\psi$ holds at every world $w^{\prime}$ with $w R_{\phi} w^{\prime}$. In other words, $\phi \rightarrow \psi$ is treated as $[\phi] \psi$ using a normal modal necessity operator $[\phi]$.

Variants of this semantics have been used, for example, to support the intensional conditional connectives of Stalnaker and Thomason [28, 29] and Priest [22] who write it with the notation >, Lewis [19] who writes it as $\square \longrightarrow$ and Chellas [4] who writes it as $\Rightarrow$.

Many propositional logics feature a pair $(\rightarrow, \triangleright)$ of connectives related by an admissible bi-directional proof rule

(RCCADJ)

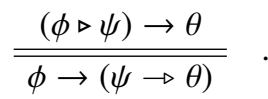

Semantically, the connectives $\triangleright$ and $\rightarrow$ are matched in an adjoint relationship. The connective $\rightarrow$ is then often thought of as a form of conditional, and the connective $\triangleright$ as a conjunction. This holds, in particular, between material implication and conjunction in classical logic, intuitionistic implication and conjunction in intuitionistic logic, relevant implication and the fusion connective of various relevant/substructural logics, and the multiplicative/linear lollipop and tensor of linear logics.

No matching conjunction is usually made explicit for the conditional connective appearing in conditional logics. This may be because such a connective is not seen to be useful, nor to suggest straightforward applications. However, a matching partner to the conditional connective $\rightarrow$ is supported by the simple semantics above. Let $R_{\psi}^{-1}$ be the usual inverse relation to $R_{\psi}$, and let $\langle\psi\rangle^{-1}$ be the corresponding normal modal possibility operator. Defining $\phi \triangleright \psi$ to be interpreted in the same way as $\langle\psi\rangle^{-1} \phi$ makes the rule RCCADJ sound. A similar thing could be done with many other logics featuring implications modelled as propositionally indexed families of normal modal necessity operators. In this paper, conditional logics with both $\rightarrow$ and $\triangleright$ are studied. The name recension is used for the connective $\triangleright$. 
Unpacking the semantic definitions, and using the notation $\models_{w}^{(\mathcal{W}, v)} \phi$ for the satisfaction of a formula $\phi$ at world $w$ on a frame $\mathcal{W}$ with worlds $W$ and binary relations $R_{\phi}$ (indexed by formulae, as above) and valuation $v$ of atomic formulae as subsets of $W$, there is the following interpretation of the conditional:

$$
\models_{w}^{(\mathcal{W}, v)} \phi \rightarrow \psi \text { iff, for all } w^{\prime} \in W, w R_{\phi} w^{\prime} \text { implies } \models_{w^{\prime}}^{(\mathcal{W}, v)} \psi .
$$

For the new connective:

$$
\models_{w^{\prime}}^{(\mathcal{W}, v)} \phi \triangleright \psi \text { iff, there is } w \in W \text { such that } w R_{\psi} w^{\prime} \text { and } \models_{w}^{(\mathcal{W}, v)} \phi .
$$

The connective $\triangleright$ can be thought of as a form of conditional conjunction. A reading of it in natural language is perhaps less straightforward than for the conditional, where we understand a formula $\psi \rightarrow \theta$ to hold at a world $w$ as if $\psi$, then $\theta$ holding in the actual state of affairs $w$, and where this is justified by consideration of some hypothetical or fictional worlds $w^{\prime}$ reached from $w$ under hypothesis $w$. A famous example of this type reads 'if kangaroos had no tails, then they would topple over' However, we can often give $\phi \triangleright \psi$ a reading $\phi$ and yet, hypothetically, $\psi$. We think of this as holding at worlds $w^{\prime}$ that could arise under hypothesis $\psi$ from some state of affairs $w$ satisfying $\phi$. That is, $\phi \triangleright \psi$ is understood, informally, as something that may hold of certain possible worlds that can be imagined (under a hypothesis) from certain others. The right conjunct contains the hypothesis. The left conjunct provides a constraint on the world $w^{\prime}$ satisfying $\phi \triangleright \psi$, in that some world $w$ satisfying $\phi$ must be $\psi$-related to $w^{\prime}$. Returning to the kangaroo example, in the hypothetical world $w^{\prime}$ in which they have no tails but which is otherwise the same as this one, the recension allows us to express, of some useful model on frames, that 'unstable objects topple over, and yet hypothetically kangaroos have no tails' holds, but to have that 'kangaroos are fish, and yet hypothetically kangaroos have no tails' does not hold of $w^{\prime}$.

The connective $\triangleright$ is non-commutative in general, although there are many important special cases in which it is commutative. Moreover, the right conjunct can, in some models, update and override the left conjunct. For example, in some models some worlds may satisfy $\phi \triangleright(\neg \phi)$ for some formulae $\phi$. There are other well-known connections between logics with belief revision and conditionality [10].

In this paper, a logic CCK is introduced, featuring the recension connective. CCK is a conservative extension of the well-known conditional logic CK of Chellas [4].

A number of applications of the recension connective are given towards the end of the paper. We introduce these briefly now.

Firstly, the principle of augmentation $(\phi \rightarrow \psi) \rightarrow\left(\left(\phi^{\prime} \wedge \phi\right) \rightarrow \psi\right)$, that is not sound on all frames, but is of interest in many applications, can be weakened to a rule

(RCCAUG)

$$
\frac{\phi \rightarrow \psi}{\left(\phi^{\prime} \triangleright \phi\right) \rightarrow \psi}
$$

when the identity principle $\phi \rightarrow \phi$ holds.

Secondly, the semantics of the conditional and recension can be shown to include the semantics of substructural (multiplicative) connectives occurring in logics supported by a ternary relation. Important special cases of this deal with relations describing the combination of various kinds of computational resource. The multiplicative implication naturally describes properties that hold if more resource is added, while the multiplicative conjunction describes situations in which a resource can be split into two so that the constituents satisfy the conjuncts in a certain way. The conditional and recension are more general, and also give access to resource reasoning principles that cannot be described with the usual multiplicative connectives. They allow one to describe properties that hold of worlds that are related in more general ways. A simple example is given in Section 5.

A third application for the recension connective is in the treatment of contrary-to-duty problems in Deontic logic. The recension connective makes it possible to describe contrary-to-duty states of affairs in which a formula (with unary obligation modality $O$ ) such as $(O(p)) \triangleright(\neg p)$ holds, in which $p$ is supposed to be obligatory, and yet $p$ does not hold. 
Finally, a particularly important application of conditional reasoning is in the context of strategic interaction, particularly as formalised through game theory. For extensive form games consisting of sequences of moves by players, it is common for a player to reason that if play reached a certain stage, then it would continue in a certain way. It is known that the form of conditional reasoning implicit in such sentences is often critical in situations where there are epistemic considerations regarding knowledge of players about the rationality of other players. The recension connective enables us to express properties of hypothetical situations in which a player is rational (in a certain sense), and yet, play reaches a state that could not have been reached if the player had been rational from the beginning of the game.

The remainder of the paper is structured as follows. Section 2 defines the logic CCK of interest. Section 3 gives a sound and complete algebraic semantics. Section 4 gives a semantics in terms of binary relational frames, extending the semantics of conditional logic given by Chellas [4]. Section 5 looks at applications of the recension connective. Section 6 demonstrates completeness results for CCK. It also shows how to adapt the usual method of filtration to apply to the situation with the recension connective together with the conditional; this allows us to infer the existence of a complete finite model, and from that the decidability of CCK. Extensions of CCK with standard axioms from Lewis's famous VC also have complete finite models because the Strevens filtration technique [26] continues to work in the presence of the recension connective. Section 7 shows how to define the semantics of the recension connective in terms of (Scott-Montague) minimal/neighourhood models. Section 8 studies the relationship between the conditional and recension of CCK and the implications and multiplicative conjunction (fusion), respectively, of substructural and relevant logics that use a ternary relation semantics to model these connectives. Section 9 gives a sound and complete calculus using world-labelled tableau. Section 10 discusses further work.

\section{LANGUAGE AND LOGIC}

Let Var be a denumerable set of propositional variables (atomic sentences). The basic language, $\mathcal{L}(\rightarrow$ $, \triangleright)$, is the set of well-formed propositional formulae formed from the constant $\perp$ (falsity), by closing under the binary operations $\rightarrow$ ((truth-functional/material) implication), $\rightarrow$ (conditional) and $\triangleright$ (conditional conjunction or recension). Let the set of propositional formulae be Form. The letters $\phi, \psi, \theta, \ldots$ are used to range over propositions.

Throughout the paper we are interested in extending classical boolean propositional logic. Thus the constants $\top$ (truth), and the usual operations $\neg$ (negation), $\wedge$ (conjunction), $\vee$ (disjunction), $\leftrightarrow$ (bi-implication) will be definable in the usual way.

A logic is a set of formulae, called theorems, that contains all (classical) truth-functional tautologies, is closed under the rule of modus ponens, and is closed under substitution of formulae for theorems. When the logic is over the basic language, this may be referred to as a conditional conjunction logic or a conditional logic with recension.

For any formula $\phi$ and logic $L$, we write $r_{L} \phi$ if $\phi \in L$. If $L$ is a logic and $\Gamma$ is a set of formulae, then we write $\Gamma \vdash_{\mathrm{L}} \phi$ if there are $\phi_{1}, \ldots, \phi_{n} \in \Gamma$ such that $\phi_{1} \wedge \ldots \wedge \phi_{n} \rightarrow \phi \in \mathrm{L}$. In this case, $\phi$ is derivable from $\Gamma$ in $\mathrm{L}$. We write $\Gamma \nvdash_{L} \phi$ just if it is not the case that $\Gamma \vdash_{L} \phi$. The relation $\phi \vdash_{L} \psi$ holds when $\phi \vdash_{L} \psi$ and $\psi \vdash_{L} \phi$ both hold. When the logic, L, is clear from the context, we may drop the subscript on these relations.

The inference rule notations

$$
\frac{\phi}{\psi} \quad \text { and } \quad \frac{\phi}{\bar{\psi}}
$$

are used, respectively, for rules of the form 'if $r_{L} \phi$ then $r_{L} \psi$ ', and ' $r_{L} \phi$ if and only if $r_{L} \psi$ '.

A conditional logic is a logic for the basic language but excluding the recension connective $\triangleright$. A conditional logic is normal [4] if it closed under the rules RCEA and RCK shown in Table 1. This can be alternatively characterized using RCEA, RCEC and the monotonicity, regularity and normality axioms, CM, $\mathrm{CR}^{1}$ and $\mathrm{CN}$, respectively. The logic $\mathrm{CK}$ is the smallest normal conditional logic.

Table 2 lists a handful of important possible rules for conditional logics with recension. There are a number of easy relations between rules given in Proposition 2.1.

\footnotetext{
${ }^{1}$ Chellas calls this CC.
} 
(RCEA) If $\vdash \mathrm{L} \phi \leftrightarrow \phi^{\prime}$, then $\vdash_{\mathrm{L}}(\phi \rightarrow \psi) \leftrightarrow\left(\phi^{\prime} \rightarrow \psi\right)$.

(RCEC) If $\vdash_{L} \psi \leftrightarrow \psi^{\prime}$, then $\vdash_{L}(\phi \rightarrow \psi) \leftrightarrow\left(\phi \rightarrow \psi^{\prime}\right)$.

(RCM) If $\vdash_{L} \psi \rightarrow \psi^{\prime}$, then $\vdash_{L}(\phi \rightarrow \psi) \rightarrow\left(\phi \rightarrow \psi^{\prime}\right)$.

(RCR) If $\vdash_{\mathrm{L}} \psi \wedge \psi^{\prime} \rightarrow \theta$, then $\vdash_{\mathrm{L}}\left((\phi \rightarrow \psi) \wedge\left(\phi \rightarrow \psi^{\prime}\right)\right) \rightarrow(\phi \rightarrow \theta)$.

(RCK) If $\vdash_{\mathrm{L}}\left(\psi_{1} \wedge \ldots \wedge \psi_{n}\right) \rightarrow \psi$, then $\vdash_{\mathrm{L}}\left(\left(\phi \rightarrow \psi_{1}\right) \wedge \ldots \wedge\left(\phi \rightarrow \psi_{n}\right)\right) \rightarrow(\phi \rightarrow \psi)$.

(CM) $\quad \vdash_{\mathrm{L}}\left(\phi \rightarrow\left(\psi \wedge \psi^{\prime}\right)\right) \rightarrow\left((\phi \rightarrow \psi) \wedge\left(\phi \rightarrow \psi^{\prime}\right)\right)$

(CR) $\quad \vdash_{\mathrm{L}}\left((\phi \rightarrow \psi) \wedge\left(\phi \rightarrow \psi^{\prime}\right)\right) \rightarrow\left(\phi \rightarrow\left(\psi \wedge \psi^{\prime}\right)\right)$

(CN) $\quad r_{\mathrm{L}} \phi \rightarrow \mathrm{T}$

TABLE 1. Rules and Axioms for Normal Conditional Logics

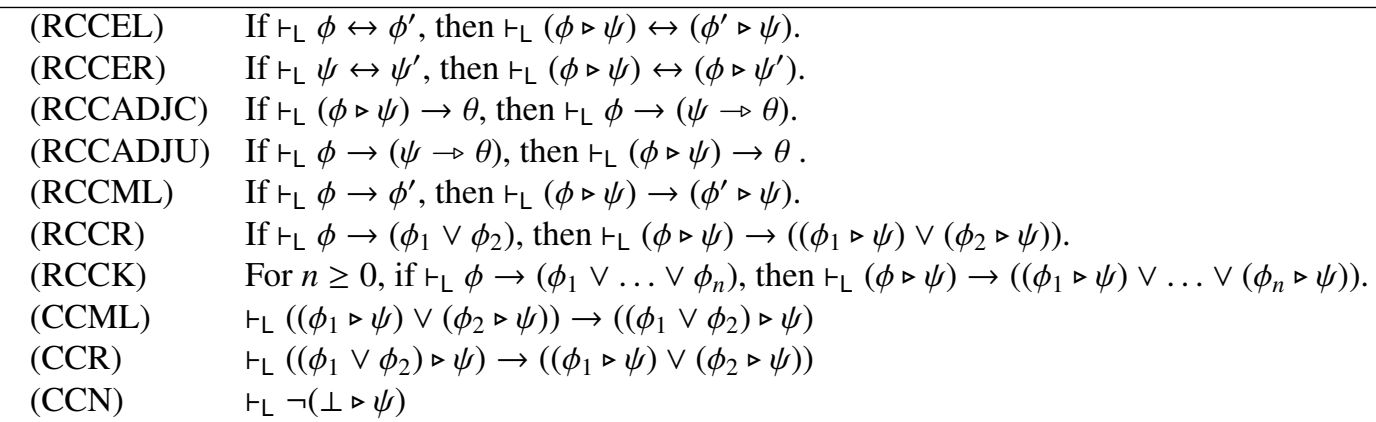

TABLE 2. Possible Rules and Axioms for Conditional Logics with Recension

Proposition 2.1. For any logic over the basic language:

(1) RCCML is derivable from RCCADJC, RCCADJU;

(2) RCCEL is derivable from RCCML;

(3) RCEA is derivable from RCCER, RCCADJC, RCCADJU;

(4) $R C E C$ is derivable from RCM;

(5) $R C M$ is derivable from RCCADJC, RCCADJU;

(6) $C C M L$ is derivable from $R C C M L$;

(7) $C C N$ is derivable from RCCADJU;

(8) $C C R$ is derivable from $R C C R$;

(9) $R C C R$ is derivable from $C C R, R C C M L$;

(10) RCCR is derivable from RCCADJC, RCCADJU;

(11) $R C C K$ is derivable from RCCML, RCCR;

(12) $R C K$ is derivable from RCCML, RCCADJC and RCCADJU.

Proof. Proofs for a few essential cases are indicated below. Only instances of assumptions, axioms and propositional tautologies for classical propositional logic are not labelled in derivations.

(1) This is demonstrated via the derivation

$$
\frac{\vdash_{\mathrm{L}} \phi \rightarrow \phi^{\prime} \quad \frac{\vdash_{\mathrm{L}}\left(\phi^{\prime} \triangleright \psi\right) \rightarrow\left(\phi^{\prime} \triangleright \psi\right)}{\vdash_{\mathrm{L}} \phi^{\prime} \rightarrow\left(\psi \rightarrow\left(\phi^{\prime} \triangleright \psi\right)\right)}}{\frac{\vdash_{\mathrm{L}} \phi \rightarrow\left(\psi \rightarrow\left(\phi^{\prime} \triangleright \psi\right)\right)}{\vdash_{\mathrm{L}}(\phi \triangleright \psi) \rightarrow\left(\phi^{\prime} \triangleright \psi\right)} \text { RCCADJC }}
$$

(3) The desired bidirectional implication is derived via a pair of derivations, one for each direction of the implication, and each of which is a very and obvious minor modification of the other. One of the pair is 


$$
\operatorname{RCCER} \frac{\vdash_{\mathrm{L}} \phi \leftrightarrow \phi^{\prime}}{\frac{\vdash_{\mathrm{L}}\left(\left(\phi^{\prime} \rightarrow \psi\right) \triangleright \phi\right) \leftrightarrow\left(\left(\phi^{\prime} \rightarrow \psi\right) \triangleright \phi^{\prime}\right)}{\vdash_{\mathrm{L}}\left(\left(\phi^{\prime} \rightarrow \psi\right) \triangleright \phi\right) \rightarrow\left(\left(\phi^{\prime} \rightarrow \psi\right) \triangleright \phi^{\prime}\right)}} \quad \frac{\vdash_{\mathrm{L}}\left(\phi^{\prime} \rightarrow \psi\right) \rightarrow\left(\phi^{\prime} \rightarrow \psi\right)}{r_{\mathrm{L}}\left(\left(\phi^{\prime} \rightarrow \psi\right) \triangleright \phi^{\prime}\right) \rightarrow \psi} \text { RCCADJU }
$$

(5) This is shown by the derivation

$$
\operatorname{RCCADJU} \frac{\vdash_{\mathrm{L}}(\phi \rightarrow \psi) \rightarrow(\phi \rightarrow \psi)}{\frac{\vdash_{\mathrm{L}}((\phi \rightarrow \psi) \triangleright \phi) \rightarrow \psi}{\operatorname{RCCADJC} \frac{\vdash_{\mathrm{L}}((\phi \rightarrow \psi) \triangleright \phi) \rightarrow \psi^{\prime}}{\vdash_{\mathrm{L}}(\phi \rightarrow \psi) \rightarrow\left(\phi \rightarrow \psi^{\prime}\right)}}}
$$

(12) For each $1 \leq i \leq n$, using the abbreviation $\mathrm{r}$ for RCCADJU, there is a derivation

$$
\operatorname{RCCML} \frac{\vdash_{\mathrm{L}}\left(\left(\phi \rightarrow \psi_{1}\right) \wedge \ldots \wedge\left(\phi \rightarrow \psi_{n}\right)\right) \rightarrow\left(\phi \rightarrow \psi_{i}\right)}{\frac{\vdash_{\mathrm{L}}\left(\left(\left(\phi \rightarrow \psi_{1}\right) \wedge \ldots \wedge\left(\phi \rightarrow \psi_{n}\right)\right) \triangleright \phi\right) \rightarrow\left(\left(\phi \rightarrow \psi_{i}\right) \triangleright \phi\right)}{\vdash_{\mathrm{L}}\left(\left(\left(\phi \rightarrow \psi_{1}\right) \wedge \ldots \wedge\left(\phi \rightarrow \psi_{n}\right)\right) \triangleright \phi\right) \rightarrow \psi_{i}} \quad \frac{\vdash_{\mathrm{L}}\left(\phi \rightarrow \psi_{i}\right) \rightarrow\left(\phi \rightarrow \psi_{i}\right)}{\left.\vdash_{\mathrm{L}}\left(\left(\phi \rightarrow \psi_{i}\right) \triangleright \phi\right) \rightarrow \psi_{i}\right)}} \mathrm{r}
$$

We can therefore derive

$$
r_{\mathrm{L}}\left(\left(\left(\phi \rightarrow \psi_{1}\right) \wedge \ldots \wedge\left(\phi \rightarrow \psi_{n}\right)\right) \triangleright \phi\right) \rightarrow\left(\psi_{1} \wedge \ldots \wedge \psi_{n}\right)
$$

and from this we can make the derivation

$$
\frac{\vdash_{\mathrm{L}}\left(\left(\left(\phi \rightarrow \psi_{1}\right) \wedge \ldots \wedge\left(\phi \rightarrow \psi_{n}\right)\right) \triangleright \phi\right) \rightarrow\left(\psi_{1} \wedge \ldots \psi_{n}\right) \quad \vdash_{\mathrm{L}}\left(\psi_{1} \wedge \ldots \wedge \psi_{n}\right) \rightarrow \psi}{\operatorname{RCCADJC} \frac{\vdash_{\mathrm{L}}\left(\left(\left(\phi \rightarrow \psi_{1}\right) \wedge \ldots \wedge\left(\phi \rightarrow \psi_{n}\right)\right) \triangleright \phi\right) \rightarrow \psi}{\vdash_{\mathrm{L}}\left(\left(\phi \rightarrow \psi_{1}\right) \wedge \ldots \wedge\left(\phi \rightarrow \psi_{n}\right)\right) \rightarrow(\phi \rightarrow \psi)}}
$$

Any logic that is closed under the rules RCCER, RCCADJC, RCCADJU is said to be basal. Such a logic is monotonic, in the sense that RCM holds and basic $^{2}$ in the sense that RCEA and RCEC both hold. The logics discussed below will all be basal, unless explicitly stated to the contrary, except in Section 9. Every basal logic satisfies the following congruence condition:

Theorem 2.2 (Replacement of Provable Equivalents). Let $\phi$ be a formula. Let $\psi$ be a formula occuring at a particular position in $\phi$ and let $\phi^{\prime}$ be the formula formed from $\phi$ by replacing just that occurrence of $\psi$ with another formula $\psi^{\prime}$. Then $\phi \dashv \phi^{\prime}$ if $\psi \dashv \psi^{\prime}$.

Proof. The proof is by induction on the complexity (height of the syntax tree) of $\phi$. See Segerberg [26] for all cases of the induction except for those involving the recension connective. In particular, the induction step for $\rightarrow$ goes through because we still have RCEA and RCEC. The induction step for $\triangleright$ goes through because we have RCCEL and RCCER, with details as below.

Consider a formula $\phi$ with a subformula $\psi$ occurring in a particular position such that the outermost (that is, leftmost, when rewritten in Polish notation) connective of $\phi$ is the recension connective, and suppose that $\phi^{\prime}$ is the formula that results from replacing the same occurrence of $\psi$ with $\psi^{\prime}$ such that $\psi \dashv \psi^{\prime}$. By the induction hypothesis (that the theorem holds for all formulae of lower complexity than $\phi$ ), it suffices to consider the cases where $\psi$ is the immediate left- or right-recension-conjunct of $\phi$. First, note that $\theta \dashv \theta^{\prime}$ if and only if $\vdash \theta \leftrightarrow \theta^{\prime}$ for any two formulae $\theta, \theta^{\prime}$. For the left-conjunct case, let $\phi$ be $\psi \triangleright \theta$, so that $\phi^{\prime}$ is $\psi^{\prime} \triangleright \theta$. Applying RCCEL gives $\psi \triangleright \theta \dashv \psi^{\prime} \triangleright \theta$ as required. For the right-conjunct case, let $\phi$ be $\theta \triangleright \psi$, so that $\phi^{\prime}$ is $\theta \triangleright \psi^{\prime}$. Applying RCCER gives $\theta \triangleright \psi \dashv t \theta \triangleright \psi^{\prime}$ as required.

A normal logic for $\mathcal{L}(\rightarrow, \triangleright)$ is one that is closed under the RCK, RCCER, RCCADJC, RCCADJU rules. A normal logic is double-normal if RCCK also holds. Proposition 2.1 shows that every basal logic is double-normal. Let the smallest such logic be CCK.

\footnotetext{
${ }^{2}$ Terminology follows Segerberg [26]. Chellas [4] calls this classical, mirroring terminology from Lemmon and Scott [18].
} 
As Chellas [4] observed, the rules RCEA and RCEC allow the treatment of propositional formulae $\psi \rightarrow \theta$ as though they were relative necessity modalities of the form $\left.\left[{ }^{2}\right]_{\mathrm{L}}\right] \theta$, where $[\psi]_{\mathrm{L}}$ is the equivalence class of $\psi$ under $\dashv \mathrm{L}$. The conditions for this modality to be normal are then just the conditions for CK for conditional logic. The rules RCCEL and RCCER allow $\triangleright$ to be regarded as a family of possibility modalities, with $\phi \triangleright \psi$ as $\left\langle[\psi]_{L}^{-1}\right\rangle \phi$. The inversion notation, -1 , signifies that the notion of possibility is inverted, and in the binary relational frame semantics the relation is inverted. The normality condition for this operator is just RCCK. Basal logics therefore allow both the conditional and recension operator to be treated as normal modal operators.

\section{Algebraic Semantics}

Define a closed magma boolean algebra (CMB-algebra) to be a Boolean algebra, $B$, with order $\leq$ and the usual structure $\perp, \top, \rightarrow, \wedge, \vee, \neg$ on the carrier set $|B|$, together with a pair of binary operations $\rightarrow$ and $\triangleright$ on $|B|$ such that

$$
b \triangleright b^{\prime} \leq b^{\prime \prime} \text { iff } b \leq b^{\prime} \rightarrow b^{\prime \prime}
$$

for all $b, b^{\prime}, b^{\prime \prime}$ in $|B|$. We will usually omit the notation for extracting the carrier set, for example writing it simply as $B$.

Structures of this type have been widely investigated and for many years [13, 30]. Recall that a magma is a structure consisting of a set together with a binary operation on that set, and that a magma operation on an ordered set is (left-)residuated when the closure condition 3 (residuation) holds. A CMB-algebra is therefore a boolean algebra and a left-residuated magma.

Left-monotonicity of $\triangleright$ is a consequence of residuation:

$$
b \leq b^{\prime} \text { implies } b \triangleright b^{\prime \prime} \leq b^{\prime} \triangleright b^{\prime \prime}
$$

for all $b, b^{\prime}, b^{\prime \prime}$ in $B$.

A CMB-algebra is collapsed if the $\rightarrow$ and $\rightarrow$ operations are identical. Examples arise commonly in computer science, see for example recent work by Collinson, McDonald and Pym [6].

Let $B$ be a boolean algebra with a pair of magma operations, $\triangleright$ and $\rightarrow$. If, for all $b, b^{\prime}, b^{\prime \prime}$ in $B$,

$$
b \rightarrow\left(b^{\prime} \wedge b^{\prime \prime}\right) \leq\left(\left(b \rightarrow b^{\prime}\right) \wedge\left(b \rightarrow b^{\prime \prime}\right)\right),
$$

then $B$ is monotonic. If $B$ is monotonic and

$$
\left(\left(b \rightarrow b^{\prime}\right) \wedge\left(b \rightarrow b^{\prime \prime}\right)\right) \leq b \rightarrow\left(b^{\prime} \wedge b^{\prime \prime}\right)
$$

then it is regular. If $B$ is regular and

$$
b \rightarrow \top=\top
$$

then it is normal. B is double-normal if it is normal and the conditions

$$
\left(b \vee b^{\prime \prime}\right) \triangleright b^{\prime}=\left(b \triangleright b^{\prime}\right) \vee\left(b^{\prime \prime} \triangleright b^{\prime}\right)
$$

and

$$
\perp \triangleright b=\perp
$$

hold.

In terms of category theory, for each $b^{\prime}$ in a CMB-algebra $B$, the assignment $F_{b^{\prime}}: b \mapsto b \triangleright b^{\prime}$ and the assignment $G_{b^{\prime}}: b^{\prime \prime} \mapsto b^{\prime} \rightarrow b^{\prime \prime}$ define functors on the category defined by the poset $B$ (with an arrow $b \rightarrow b^{\prime}$ just when $b \leq b^{\prime}$ ). The former assignment is functorial by the condition 4 on left-monotonicity of $\triangleright$. The functoriality of the latter assignment follows from the condition 3, which shows that the assignments define a family of adjunctions parametrised by $b^{\prime}$, with $F_{b^{\prime}}$ on the left. It is a general fact of category theory that right adjoints preserve all limits. Greatest lower bounds and $T$ are limits, while greatest upper bounds and $\perp$ are colimits. Therefore, all CMB-algebras are double-normal. 
A valuation of the basic language on a CMB-algebra $B$ is a function $v:$ Var $\longrightarrow B$. Each valuation corresponds to a unique interpretation of the language on $B$, written $I_{v}:$ Form $\longrightarrow B$, where this function is required to interpret the classical boolean connectives using the Boolean algebra $B$ as usual, and moreover to have $I_{v}(\phi \rightarrow \psi)=I_{v}(\phi) \rightarrow I_{v}(\psi)$ and $I_{v}(\phi \triangleright \psi)=I_{v}(\phi) \triangleright I_{v}(\psi)$. Note that RCEA, RCEC, RCCEL, RCCER mean that for a basal logic, if $\phi \dashv \phi^{\prime}$, then $I_{v}(\phi)=I_{v}\left(\phi^{\prime}\right)$. Write $\models^{(B, v)} \phi$ if $I_{v}(\phi)=\top$. A rule of the form $\frac{\phi}{\psi}$, that is 'if $\phi$, then $\psi$ ', is sound (on $B$ with $v$ ) if $\models^{(B, v)} \psi$ whenever $\models^{(B, v)} \phi$.

Proposition 3.1. The rules RCCER, RCCADJC, RCCADJU are sound on CMB-algebras. Hence, the logic CCK is sound on $C M B$-algebras: if $\operatorname{tCcK} \phi$ then $\models^{(B, v)} \phi$.

Proof. Soundness of RCCER follows the fact that the recension connective is interpreted by the magma operation. Soundness of RCCADJC and RCCADJU are guaranteed by the residuation condition 1 and because conditional is interpreted by the left residuation of the magma operation. The logic CCK is determined by just the three rules RCCER, RCCADJC, RCCADJU.

The soundness result above has the corresponding adequacy result encapsulated in the completeness theorem below.

Theorem 3.2. The logic CCK is complete on CMB-algebras: if $\models^{(B, v)} \phi$ for all CMB-algebras $B$ and

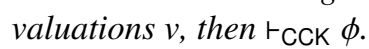

Proof. A Lindenbaum algebra, taking the form of a CMB-algebra, and canonical valuation are constructed from the logic in the usual way. The well-definedness of the operations $\triangleright$ and $\rightarrow$ follows from the rules RCCER, RCCEL, RCEA, RCEC under which CCK is closed, the final three of which we have from Proposition 2.1. The fact that the residuation condition 3 holds follow from the rules RCCADJC and RCCADJU.

\section{Frame Semantics}

\subsection{Interpretation on Ch-frames.}

A $C h$-frame is a structure $\mathcal{W}=\langle W, f\rangle$, where $W=|\mathcal{W}|$ is the carrier set and $f$ is a function $f: W \times \mathcal{P} W \longrightarrow$ $\mathcal{P} W$, where $\mathcal{P} W$ is the power set of $\mathrm{W}$. The function $f$ will be called the transition operation (or function) ${ }^{3}$.

Let $U^{\complement}$ be the complement of any subset $U$ of $W$. Let

$$
[U] U^{\prime}=\left\{w \in W \mid f(w, U) \subseteq U^{\prime}\right\}
$$

and

$$
f\left(U, U^{\prime}\right)=\bigcup_{w \in U} f\left(w, U^{\prime}\right)
$$

for all $U, U^{\prime} \subseteq W$.

Central to the interpretation of normal conditional logic on relational (Kripke-like) frames is the fact that the frame induces a suitable algebra of subsets that is closed under a modal necessity operation corresponding to the relations. In general, this need not be the Boolean algebra consisting of the whole power set, see for example Segerberg [26]. The simple, but important, result of Theorem 4.1 below shows that this approach extends to normal conditional logics with recension.

Theorem 4.1. If $\mathcal{W}=\langle W, f\rangle$ is a Ch-frame, then there is an induced CMB-algebra on $\mathcal{P} W$, with

$$
U \rightarrow U^{\prime}=[U] U^{\prime}=\left\{w \in W \mid f(w, U) \subseteq U^{\prime}\right\}
$$

and

$$
U \triangleright U^{\prime}=f\left(U, U^{\prime}\right)
$$

for all $U, U^{\prime} \subseteq W$.

\footnotetext{
${ }^{3}$ The terminology is non-standard. We use the prefix 'Ch-' to differentiate from the other frames used later in the paper. Chellas [4] calls these standard frames. It is convenient to present the $\mathcal{P} W$-indexed family of binary accessibility relations as a multifunction.
} 
Proof. The Boolean algebra structure is the usual one on the power set. The definition of the conditional operation 5 matches that implicit in Chellas [4] and explicit in Segerberg [26]. It is known (Theorem 3 [4]) and easy to check that this operation satisfies the conditions for normality. That the residuation condition 3 for a CMB-algebra holds is easily verified directly:

$$
U \triangleright U^{\prime} \subseteq U^{\prime \prime} \text { iff } U \subseteq U^{\prime} \rightarrow U^{\prime \prime}
$$

for all $U, U^{\prime}, U^{\prime \prime} \subseteq W$.

A valuation on a Ch-frame $\mathcal{W}=\langle W, f\rangle$ is simply a valuation on the induced CMB-algebra, $v:$ Var $\longrightarrow$ $\mathcal{P} W$. This corresponds uniquely to an interpretation (on the underlying CMB-algebra); this will be written $\llbracket-\rrbracket^{(\mathcal{W}, v)}:$ Form $\longrightarrow \mathcal{P} W$. A model is a pair $\mathcal{M}=(\mathcal{W}, v)$, where $\mathcal{W}$ is a frame and $v$ is a valuation.

Let $\mathcal{W}$ be a Ch-frame with carrier set $W$, let $v$ be a valuation and let $w \in W$. Define $\models_{w}^{(\mathcal{W}, v)} \phi$ if $w \in \llbracket \phi \rrbracket^{(\mathcal{W}, v)}$ (and say $\phi$ is true (or holds) at $w$ in $\mathcal{W}$ under (or with) valuation $v$ ). Define $\models^{(\mathcal{W}, v)} \phi$ if $\models_{w}^{(\mathcal{W}, v)} \phi$ for all $w$ in $\mathcal{W}$, and say $\phi$ is true on the model $\mathcal{M}=(\mathcal{W}, v)$. Define $\models^{\mathcal{W}} \phi$ if $\models^{(\mathcal{W}, v)} \phi$ for all valuations $v$, and say $\phi$ is valid in $\mathcal{W}$. Say that $\phi$ is valid if it is valid on all Ch-frames.

Let $\Gamma$ be a (possibly infinite) set of propositional formulae and $\psi$ be another propositional formula. The local semantic consequence notation $\Gamma \models_{w}^{\mathcal{M}} \psi$ at a world $w$ means that if $\models_{w}^{\mathcal{M}} \phi$ for all of the formulae $\phi$ in $\Gamma$, then $\models_{w}^{\mathcal{M}} \psi$. The notation $\Gamma \models^{\mathcal{M}} \psi$ means that $\Gamma \models_{w}^{\mathcal{M}} \psi$ for all $w$ in the carrier set of $\mathcal{M}$. The global semantic consequence notation $\Gamma \mathbb{F}^{\mathcal{M}} \psi$ means that if $\models^{\mathcal{M}} \phi$ for every $\phi$ in $\Gamma$, then $\models^{\mathcal{M}} \psi$.

We say that rule of the form $\frac{\phi}{\psi}$, that is if $\phi$ then $\psi$, is sound on a model $\mathcal{M}=(\langle W, f\rangle, v)$ if and only if it is sound on the Boolean algebra $\mathcal{P} B$ with the same valuation $v$. This is the same as $\phi \mathbb{F}^{\mathcal{M}} \psi$.

For the conditional connective we find the usual Chellas [4] interpretation:

$$
\models_{w}^{(\mathcal{W}, v)} \phi \rightarrow \psi \text { iff } f\left(w, \llbracket \phi \rrbracket^{(\mathcal{W}, v)}\right) \subseteq \llbracket \psi \rrbracket^{(\mathcal{W}, v)} .
$$

For the recension:

$$
\models_{W^{\prime}}^{(\mathcal{W}, v)} \phi \triangleright \psi \text { iff there is } w \in W \text { such that } \models_{w}^{(\mathcal{W}, v)} \phi \text { and } w^{\prime} \in f\left(w, \llbracket \psi \rrbracket^{(\mathcal{W}, v)}\right) .
$$

Thus, worlds satisfying this notion of conditional conjunction are those that could arise from worlds satisfying the left-conjunct under the action of the transition using the right-conjunct. These clauses in the interpretation should be compared to those marked 1 and 2 in Section 1. The only significant difference is in the use of a frame with relations indexed by subsets rather than formulae, and this relates to the RCEA and RCCER axioms.

Corollary 4.2 follows immediately from Theorem 4.1 and the relationship 7. A further consequence is soundness, Corollary 4.3.

Corollary 4.2. For any model, $\mathcal{M}$ and $\phi, \psi, \theta$, it is the case that $\models^{\mathcal{M}}(\phi \triangleright \psi) \rightarrow \theta$ if and only if $\models^{\mathcal{M}}$ $\phi \rightarrow(\psi \rightarrow \theta)$. That is, both of the global semantic consequences $(\phi \triangleright \psi) \rightarrow \theta \mathbb{F}^{\mathcal{M}} \phi \rightarrow(\psi \rightarrow \theta)$ and $\phi \rightarrow(\psi \rightarrow \theta) \mathbb{F}^{\mathcal{M}} \phi \rightarrow(\psi \rightarrow \theta)$ hold.

Corollary 4.3. The logic CCK is sound on Ch-frames: for any formula $\phi$, if $\vdash_{C C K} \phi$, then $\models^{\mathcal{M}} \phi$ for all models $\mathcal{M}$ on Ch-frames.

For a Ch-frame $\mathcal{W}=\langle W, f\rangle$, the function $f$ is the material transition if for any world $w \in W$ and any $U \subseteq W$ it has

$$
f(w, U)=\left\{\begin{array}{cc}
\{w\} & \text { if } w \in U \\
\emptyset & \text { otherwise. }
\end{array}\right.
$$

If $f$ is the material conditional, then the induced algebra is collapsed. The interpretation of $\rightarrow$ becomes identical to that of material implication, and the interpretation of $\triangleright$ becomes identical to that of ordinary conjunction. 
The two-way rule

(RCCADJ)

$$
\frac{(\phi \triangleright \psi) \rightarrow \theta}{\phi \phi \rightarrow(\psi \rightarrow \theta)}
$$

summarizes the sound rules RCCADJC and RCCAJU. In contrast, the axiom

$$
((\phi \triangleright \psi) \rightarrow \theta) \leftrightarrow(\phi \rightarrow(\psi \rightarrow \theta))
$$

is not valid, unlike for ordinary conjunction and material implication. The point is that in the rule RCCADJ, the formula in the upper line holds at a world after transition via the transition along $\psi$, where the formula in the lower line holds at a (possibly different) world before the transition. Soundness of the CCADJ axiom would require them both to hold at the same world, as in the pair of local semantic consequences $(\phi \triangleright \psi) \rightarrow \theta \vDash{ }^{\mathcal{M}} \phi \rightarrow(\psi \rightarrow \theta)$ and $\phi \rightarrow(\psi \rightarrow \theta) \vDash^{\mathcal{M}} \phi \rightarrow(\psi \rightarrow \theta)$. This is not what the relationship 7 in the proof of Theorem 4.1 asserts, except in special cases. A counterexample is given in Example 4.4.

Example 4.4. Let $W=\left\{w_{1}, w_{2}\right\}$. Let $U_{1}=\left\{w_{1}\right\}, U_{2}=\left\{w_{2}\right\}$. Let $f: W \times \mathcal{P}(W) \longrightarrow \mathcal{P}(W)$ be a transition function with $f(w, U)=U_{2}$ for all pairs $w, U$, except $f\left(w_{1}, W\right)=W$. This gives a Ch-frame $W=\langle W, f\rangle$. Let $p_{1}, p_{2}$ and $p_{3}$ be propositional variables, and let $v$ be any valuation with $v\left(p_{1}\right)=U_{1}, v\left(p_{2}\right)=U_{2}$, $v\left(p_{3}\right)=U_{1}$. Then $w_{1} \in \llbracket\left(p_{1} \triangleright p_{2}\right) \rightarrow p_{3} \rrbracket_{v}^{\mathcal{W}}=f\left(U_{1}, U_{2}\right)^{\complement} \cup U_{1}=U_{2}^{\complement} \cup U_{1}=U_{1}$. However, $w_{1} \notin$ $\llbracket p_{1} \rightarrow\left(p_{2} \rightarrow p_{3}\right) \rrbracket_{v}^{\mathcal{W}}=U_{1}^{\complement} \cup\left(U_{2} \rightarrow U_{1}\right)=U_{1}^{\complement} \cup\left\{w \in W \mid f\left(w, U_{2}\right) \subseteq U_{1}\right\}=U_{1}^{\complement} \cup \emptyset=U_{2}$. Thus $(\phi \triangleright \psi) \rightarrow \theta) \rightarrow(\phi \rightarrow(\psi \rightarrow \theta)$ is not valid.

Segerberg [26] gives a treatment of the correspondence theory between logical formulae and classes of frames for extensions of CK. For now, we note only that the axioms

$$
\phi \rightarrow \phi
$$

and

(MP)

$$
(\phi \rightarrow \psi) \rightarrow(\phi \rightarrow \psi)
$$

correspond, respectively, to $\mathrm{Ch}$-frames satisfying the conditions

$$
f(w, U) \subseteq U
$$

and

(mp)

$$
\text { if } w \in U \text {, then } w \in f(w, U)
$$

for all $w \in W$ and $U \subseteq W$. The proofs in Chellas [4] are unchanged in the presence of the recension connective.

4.2. Comparing CCK and CK. Before giving the technical material on completeness, which is shown in Section 6, we use the frame semantics to compare CCK with CK.

Theorem 4.5. CCK is a conservative extension of $\mathrm{CK}$.

Proof. Let $\mathcal{L}(\rightarrow)$ be the set of well-formed propositional formulae, formed from $\perp, \rightarrow$ and $\rightarrow$ and the set of propositional variables Var. That is, $\mathcal{L}(\rightarrow)$ is the language of CK.

Every Ch-frame model of CCK is a model of CK, and the interpretation of the connectives from $\mathcal{L}(\rightarrow)$ is the same in both models: they are given by the same operation on the power-set. That is, every model of CCK is a (unique) expansion of a model of CK.

Let $\phi \in \mathcal{L}(\rightarrow)$ be a theorem of CCK. Let $\llbracket \phi \rrbracket^{(\mathcal{W}, v)}$ be the interpretation of $\phi$ as a formula of CCK as a subset of a Ch-frame model $(\mathcal{W}, v)$ and let $\llbracket \phi \rrbracket_{\mathrm{CK}}^{(\mathcal{W}, v)}$ be the interpretation as a formula of CK. We have $\llbracket \phi \rrbracket^{(\mathcal{W}, v)}=\llbracket \phi \rrbracket_{\mathrm{CK}}^{(\mathcal{W}, v)}$. Write the carrier set of any Ch-frame $\mathcal{W}$ as $|\mathcal{W}|$. Using the completeness theorem for CCK (Corollary 6.5 with Theorem 6.11 below) and the completeness theorem for CK (in Chellas [4]),

$$
\begin{array}{ccl}
\phi \in \mathrm{CCK} & \text { iff } & \llbracket \phi \rrbracket^{(\mathcal{W}, v)}=|\mathcal{W}| \text { for all models }(\mathcal{W}, v) \text { of CCK } \\
& \text { iff } & \llbracket \phi \rrbracket_{\mathrm{CK}}^{(\mathcal{W}, v)}=|\mathcal{W}| \text { for all models }(\mathcal{W}, v) \text { of CK } \\
\text { iff } & \phi \in \mathrm{CK} .
\end{array}
$$


The fact that the language with recension is strictly more expressive than the language without follows from the fact that this is known to hold for the special case described in Section 8. It can also be shown directly with the following simple example.

Example 4.6. Consider the following Ch-frame, $W$. Let $W=\left\{w_{1}, w_{2}, w_{3}\right\}, U_{1}=\left\{w_{1}\right\}$ and $U_{1}^{\complement}=W \backslash U_{1}$. Define the transition function by taking $f\left(w_{1}, V\right)=\left\{w_{2}\right\}, f\left(w_{2}, V\right)=f\left(w_{3}, V\right)=\left\{w_{1}\right\}$ for every $V \subseteq W$.

Let $\mathcal{F}=\left\{\emptyset, U_{1}, U_{1}^{\complement}, W\right\}$. Then $\mathcal{F}$ is closed under all Boolean operations and also the conditional binary operation (5 from Theorem 4.1). In particular, $V \rightarrow \emptyset=\emptyset, V \rightarrow W=W, V \rightarrow U_{1}=U_{1}^{\complement}, V \rightarrow U_{1}^{\complement}=U_{1}$. Therefore, if all atoms of $\mathcal{L}(\rightarrow)$ are valued in $\mathcal{F}$ under a valuation $v$, then the set $U_{2}=\left\{w_{2}\right\}$ is not definable. That is, there is no formula $\phi \in \mathcal{L}(\rightarrow)$ such that $\llbracket \phi \rrbracket_{\mathrm{CK}}^{(\mathcal{W}, v)}=U_{2}$. In particular, this is the case for the valuation that maps all atoms to $U_{1}$.

On the other-hand, using the binary operation for recension (6 from Theorem 4.1), $U_{1} \triangleright V=U_{2}$ for all $V$. In particular, for any of the atoms $\phi$ such that $v(\phi)=U_{1}$, it is the case that $\llbracket \phi \triangleright \phi \rrbracket^{(\mathcal{W}, v)}=U_{2}$. Thus $U_{2}$ is definable in $\mathcal{L}(\rightarrow, \triangleright)$ with any valuation that maps an atom to $U_{1}$.

\section{Applications}

\subsection{Augmentation.}

The usual principle of augmentation takes the form of an axiom

$$
(\phi \rightarrow \psi) \rightarrow\left(\left(\phi^{\prime} \wedge \phi\right) \rightarrow \psi\right)
$$

from which the rule

$$
\frac{\phi \rightarrow \psi}{\left(\phi^{\prime} \wedge \phi\right) \rightarrow \psi}
$$

is derivable. The axiom is not valid and there are models on which the rule is not sound, but it is sometimes argued that they should hold in certain applications, see Chellas [4]. However, Lemma 5.1 leads immediately to a modified augmentation rule, using recension in place of conjunction, in Proposition 5.2.

Lemma 5.1. Let $\mathcal{W}=\langle W, f\rangle$ be a Ch-frame and $v$ be a valuation, so that $\mathcal{M}=(\mathcal{W}, v)$ is a model. If $\phi \rightarrow \psi$ is true in $\mathcal{M}$, then $\left(\phi^{\prime} \triangleright \phi\right) \rightarrow \psi$ is true in $\mathcal{M}$.

If the frame satisfies the condition id, and $\left(\phi^{\prime} \triangleright \phi\right) \rightarrow \psi$ is true in $\mathcal{M}$, then the formulae $\left(\phi^{\prime} \triangleright \phi\right) \rightarrow \psi$ and $\left(\phi^{\prime} \triangleright \phi\right) \rightarrow(\psi \wedge \phi)$ are true in $\mathcal{M}$.

Proof. Suppose $\phi \rightarrow \psi$ is true in $\mathcal{M}$.. Suppose that $w^{\prime} \in \llbracket \phi^{\prime} \triangleright \phi \rrbracket^{\mathcal{M}}$. There is then a $w \in \llbracket \phi^{\prime} \rrbracket^{\mathcal{M}}$ such that $w^{\prime} \in f\left(w, \llbracket \phi \rrbracket^{\mathcal{M}}\right) \subseteq \llbracket \psi \rrbracket^{\mathcal{M}}$. Hence $\llbracket\left(\phi^{\prime} \triangleright \phi\right) \rrbracket^{\mathcal{M}} \subseteq \llbracket \psi \rrbracket^{\mathcal{M}}$, and $\llbracket\left(\phi^{\prime} \triangleright \phi\right) \rightarrow \psi \rrbracket^{\mathcal{M}}=W$.

For the second part, let $W=\llbracket\left(\phi^{\prime} \triangleright \phi\right) \rightarrow \psi \rrbracket^{\mathcal{M}}=\llbracket \phi^{\prime} \rightarrow(\phi \rightarrow \psi) \rrbracket^{\mathcal{M}}$. Consider any $w \in W$ and any $w^{\prime \prime} \in f\left(w, \llbracket \phi^{\prime} \triangleright \phi \rrbracket^{\mathcal{M}}\right)$. Now $w^{\prime \prime} \in \llbracket \phi^{\prime} \triangleright \phi \rrbracket^{\mathcal{M}}$ using id, so there is $w^{\prime} \in \llbracket \phi^{\prime} \rrbracket^{\mathcal{M}}$ such that $w^{\prime \prime} \in$ $f\left(w^{\prime}, \llbracket \phi \rrbracket^{\mathcal{M}}\right) \subseteq \llbracket \psi \rrbracket^{\mathcal{M}} \cap \llbracket \phi \rrbracket^{\mathcal{M}}$. Thus $f\left(w, \llbracket \phi^{\prime} \triangleright \phi \rrbracket^{\mathcal{M}}\right) \subseteq \llbracket \psi \rrbracket^{\mathcal{M}}$, and so therefore $w \in \llbracket\left(\phi^{\prime} \triangleright \phi\right) \rightarrow \psi \rrbracket^{\mathcal{M}}$ and $w \in \mathbb{[}\left(\phi^{\prime} \triangleright \phi\right) \rightarrow(\psi \wedge \phi) \rrbracket^{\mathcal{M}}$.

Proposition 5.2. The global semantic consequence relation

$$
\phi \rightarrow \psi \mathbb{F}^{\mathcal{M}}\left(\phi^{\prime} \triangleright \phi\right) \rightarrow \psi
$$

holds in all models $\mathcal{M}$ on Ch-frames satisfying id. The modified augmentation rule

(RCCAUG)

$$
\frac{\phi \rightarrow \psi}{\left(\phi^{\prime} \triangleright \phi\right) \rightarrow \psi}
$$

is sound on all such models.

First note that the non-commutativity of the recension connective is significant in the conclusion of the rule RCCAUG: the conclusion cannot be replaced with $\left(\phi \triangleright \phi^{\prime}\right) \rightarrow \psi$. Second, although the rule is valid, the biconditional $(\phi \rightarrow \psi) \leftrightarrow\left(\left(\phi^{\prime} \triangleright \phi\right) \rightarrow \psi\right)$ is not. Just as with the rule RCCADJU, there is an implicit change of world between the premise and conclusion. This limits the usefulness of the rule for semantic reasoning about particular worlds. 
5.2. Generalized resource reasoning. Conditional reasoning about resources occurs rather naturally. A familiar form of phrase in English begins "If I were a rich man".

Logics for reasoning about resources used during computations have been the subject of great interest in recent years, see for example Pym [23]. The starting point for many of these applications is a version of the ternary relation semantics based on a set of worlds, $W$, and with the relation induced from a binary (partial) operation $\circ$ on $W$ via

$$
R\left(w^{\prime}, w^{\prime \prime}, w\right) \text { iff } w^{\prime} \circ w^{\prime \prime} \text { is defined and } w^{\prime} \circ w^{\prime \prime}=w
$$

for all $w, w^{\prime}, w^{\prime \prime}$.

We know, in particular from Section 8, that the interpretation of substructural implication and conjunction supported by this relation is an instance of the more general conditional semantics. However, we can also find interesting, and potentially useful, resource logics with interpretations that do not satisfy the representability condition.

Let $W=\mathbb{N}=\{0,1,2, \ldots\}$ be the set of non-negative integers. Define a transition $f$ on $W$ by

$$
f(m, U)= \begin{cases}\{m\} & \text { if } m \in U \\ \left\{n \in U\left|\forall n^{\prime} \in U .\right| m-n|\leq| m-n^{\prime} \mid\right\} & \text { otherwise }\end{cases}
$$

using the absolute value notation $|-|$. This is easily verified to not be representable.

Consider a propositional letter $\phi_{\geq n}$ valued so that $v\left(\phi_{\geq n}\right)=\{m \mid m \geq n\}$. Using this letter as an antecedent to a conditional allows us to express sentences of the form "if there were at least $n$ units of resource, then ...". For example, if $\psi$ is $\phi_{n_{1}}>\phi_{n_{2}}$, then $\phi_{\geq n} \rightarrow \psi$ expresses the fact that if there were at least $n$ units of resource, then it could be split into worlds with at least $n_{1}$ and $n_{2}$ units of resource. In contrast, when $\rightarrow$ is interpreted using addition on the natural numbers as the binary operation $\circ$, a formula $\phi_{n} \rightarrow \psi$ expresses the fact that $\psi$ holds when $n$ additional units of resource are added.

The formula $\theta \triangleright \phi_{\geq n}$ says that there are at least $n$ units of resource and $\theta$ holds, or there are exactly $n$ units of resource and $\theta$ holds for some world with strictly fewer than $n$ units of resource. For example, if $\theta$ is a letter valued so that it holds of the minimum level of resource required for some action to happen (the price), the formula expresses those situations in which there are at least $n$ units of resource and sufficient resource to match the price.

Reasoning about resources is essential in the theory of concurrency. Lamport [16] surveys many of the key ideas in the field. For example, concurrent processes can starve and fail to progress if they cannot access a resource, they can deadlock because of an inability to share resources in a way necessary to allow progress, and they can be used to enforce points of synchronization as in producer-consumer systems. Lamport notes that there appears to be a fork in approaches to modelling concurrency, between approaches that focus on resource and execution state (commonly used for modelling concurrent programs directly rather than via indirect encodings, and allowing for use of invariants in proving properties of looping programs) and event-based approaches including process calculi. An exception to this is the calculus SCRP, that, in particular, allows one to straightforwardly prove invariants of state in a process framework. The reader is referred to Collinson and Pym [7] for details.

In the SCRP calculus one has system states consisting of pairs of the form $R, E$ where $R$ is a resource context (a collection of basic resources), and $E$ is a process. For example, $R$ could be a function from a set of atomic resource names $\left\{r_{1}, r_{2}, \ldots\right\}$ to the non-negative integers: call this the basic resource model. There is required to be a monoidal (partial) composition operation on the set of resources: we will then sometimes be able to find $R=R_{1} \circ R_{2}$, for example. In the basic resource model, this composition is total, and adds the values associated to each resource name: $\left(R_{1} \circ R_{2}\right)(r)=R_{1}(r)+R_{2}(r)$. A process is a syntactically-constructed entity that, given a resource context, constrains and defines how the system can evolve via a given set of actions. In particular, a process can be a concurrent composite of other processes, for example $E=E_{1} \times E_{2}$. The properties of the operation $\times$ allow us also to consider a partial monoid on the set of states with a composition given by $\left(R_{1}, E_{1}\right) \circ\left(R_{2}, E_{2}\right)=\left(R_{1} \circ R_{2}, E_{1} \times E_{2}\right)$, where equality here is Kleene-equality (each side is defined just if the other is also).

Formally, the description of the dynamics is done with a structural operational semantics generating discrete transitions of the form $R, E \stackrel{a}{\longrightarrow} R^{\prime}, E^{\prime}$, taking one state to another along an action $a$. There is thus structure present to allow modal description of the evolution of state with labelled action modalities, but also to use the multiplicative conjunction and implication to describe the splitting and combining of system 
state, thus allowing for a natural and direct logical description of fundamental questions of use of resource in concurrency (for example, starvation, deadlocks, and synchronization using resources). This logic is called MBI.

Suppose that the process $a_{1}: E_{1}$ is a process in which $n$ units of atomic resource $r_{1}$ are required in order for is to proceed and become the process $E$. In particular then,

$$
\models_{R, a_{1}: E_{1}}\left\langle a_{1}\right\rangle \top \quad \text { iff } \quad R\left(r_{1}\right) \geq n,
$$

where $\left\langle a_{1}\right\rangle$ is the standard possibility modal operator associated with performing the action $a_{1}$.

The logic MBI also has two non-standard modalities with the semantics

$$
\begin{array}{ll}
\models_{R, E}\langle a\rangle_{\nu} \phi & \text { iff } \quad \text { there exists } R^{\prime} \text { such that } \models_{R \circ R^{\prime}, E}\langle a\rangle \phi \\
\models_{R, E}[a]_{\nu} \phi & \text { iff } \quad \text { for all } R^{\prime}, \text { if } R \circ R^{\prime} \text { is defined then } \models_{R \circ R^{\prime}, E}[a] \phi
\end{array}
$$

where $[a]$ is the standard necessity modality associated with performing the action $a$. Using the basic resource model this allows us to express, for example, that a process (such as $a_{1}: E_{1}$ above) can be equipped with sufficient resource to perform an action.

We may want to say what would happen if the resource context of a state is changed in a particular way. For example, with a state $R_{1}, a_{1}: E_{1}$ with $a_{1}$ as above and $R_{1}\left(r_{1}\right)=n-2$, we know that if 2 units of $r_{1}$ were added then the action $a$ could fire and the system could progress.

For hypothetical propositions that express a change of resource context, there is an obvious way to add a modification function (in the sense of conditional semantics in this paper, not in the sense of SCRP) for hypotheses that express a change of resource context. We simply define $f\left((R, E), \phi_{S}\right)=\{(S, E)\}$, where $\phi_{S}$ is such that $\models_{R, E} \phi_{S}$ iff $R=S$. We can now consider the use of conditional connectives $\phi_{S} \rightarrow$ and $\triangleright \phi_{S}$. These give a different way of considering a change of resource context from the \langle\rangle$_{v}$ and []$_{v}$ modalities.

Continuing with $R_{1}$ and $a_{1}$ from above, let $S_{1}$ be the resource context that is the same as $R_{1}$, except that $r_{1}$ has exactly $n$ units of resource. We can then express the fact that the state satisfies the property "if the resource context were $S_{1}$, then the process $a_{1}: E_{1}$ could progress" with $\models_{R_{1}, a_{1}: E_{1}} \phi_{S_{1}} \rightarrow\left\langle a_{1}\right\rangle \mathrm{T}$.

Similarly, while we have that $S_{1}, a_{1}: E_{1}$ can progress, there are resource contexts such that it will not. That is, $\models_{S_{1}, a_{1}: E_{1}}\left\langle a_{1}\right\rangle \top$ and $\models_{S_{1}, a_{1}: E_{1}}\left(\neg\left(\left\langle a_{1}\right\rangle T\right)\right) \triangleright \phi_{S_{1}}$. This stands in contrast to the situation for a process such as $1: E_{1}$ that uses the tick action 1 that requires no resource.

\subsection{Contrary-to-duty obligations.}

Contrary-to-duty paradoxes have been the source of much discussion in deontic logic, and are these days of great significance in the computer science field of engineering multi-agent systems. However, there are notorious problems in formalization, particularly relating to inferred obligations. A famous example is Chisholm's paradox, which presents four sentences:

(1A1) It ought to be that Jones goes to the assistance of his neighbors

(1B1) It ought to be that if he does go he tells them he is coming

(1C1) If he does not go, then he ought not to tell them he is coming

(1D1) He does not go.

Here, 1A1 and 1B1 are suggestive of an inference (by Deontic Deontic Detachment (DDD), following the terminology of Saint Croix and Thomason [24]) that Jones ought to tell his neighbours that he is coming, while 1C1 and 1D1 are suggestive of an inference (by Factual Deontic Detachment (FDD)) that he ought not tell them he is coming; it is usually thought contradictory that there is an obligation for one statement and its negation. Yet these four statements seem meaningful in an everyday sense, with 1C1 understood as applying in a state of affairs that is contrary to the duty set out in 1A1. In particular, in Standard Deontic Logic (SDL), in which "ought" is read as a unary, normal modality, the formalization scrutinized is often

(1A2) $O(g)$

(1B2) $O(g \rightarrow t)$

$(1 \mathrm{C} 2) \quad(\neg g) \rightarrow O(\neg t)$

(1D2) $\neg g$.

In particular $O(t)$ and $O(\neg t)$ may be inferred, but this is specically excluded by an axiom of SDL. 
One proposal that has been advanced several times in the past, is to read one or other of the conditionals in $1 \mathrm{~B} 1$ and $1 \mathrm{C} 1$, not as material implications, but as intensional connectives of the sort considered in the present paper. This has been done, for example by Mott [20] using a formalization in a form equivalent to

(1A3) $O(g)$

(1B3) $g \rightarrow O(t)$

$(1 \mathrm{C} 3) \quad(\neg g) \rightarrow O(\neg t)$

(1D3) $\neg g$.

where $\rightarrow$ is the Lewis $\square \rightarrow$, and a similar thing could be done in an extension of CCK.

We may consider the initiation of obligations in contrary-to-duty situations in a different way using the recension connective. Suppose that the situation is to be described with a model $\mathcal{M}$ on a Ch-frame. Suppose that a world $w$ captures the present state of affairs. It may be that $O(g)$ holds in the present state, so

$$
\vDash_{w}^{\mathcal{M}} O(g) \text {. }
$$

We may wish to consider whether a state of affairs occurs such that the formula $\neg g$ holds. Then in some related world, $w^{\prime} \in f\left(w, \llbracket \neg g \rrbracket^{\mathcal{M}}\right)$, we have $O(g) \triangleright \neg g$ : that is

$$
\models_{w^{\prime}}^{\mathcal{M}} O(g) \triangleright \neg g
$$

where this is intended to capture the fact that $w^{\prime}$ is an imagined state of affairs that is contrary to the obligation that holds in the base world $w$. That is, $g$ ought to be obligatory, and yet $g$ does not hold. Let $t^{\prime}$ say 'Jones tells he is not going.' The formula $(O(g) \triangleright \neg g) \rightarrow O\left(t^{\prime}\right)$ can be used to describe a contrary-to-duty obligation, at least in part. For if

$$
\models_{w^{\prime}}^{\mathcal{M}}(O(g) \triangleright \neg g) \rightarrow O\left(t^{\prime}\right)
$$

then we may conclude $\models_{w^{\prime}}^{\mathcal{M}} O\left(t^{\prime}\right)$ and this would not contradict $\models_{w}^{\mathcal{M}} O\left(\neg t^{\prime}\right)$ were we able to infer that. Note that for 8 it is sufficient, but not necessary, that $\models_{w}^{\mathcal{M}} O(g) \rightarrow\left((\neg g) \rightarrow O\left(t^{\prime}\right)\right)$. Thus it is also sufficient, but not necessary, that $\models_{w}^{\mathcal{M}}(\neg g) \rightarrow O\left(t^{\prime}\right)$. The formula $(\neg g) \rightarrow O\left(t^{\prime}\right)$ replaces $\rightarrow$ with $\rightarrow$ in $1 \mathrm{C} 2$. We may know that 8 holds, perhaps because $\models^{\mathcal{M}}(O(g) \triangleright \neg g) \rightarrow O\left(t^{\prime}\right)$. However, this would be equivalent to knowing $\models^{\mathcal{M}} O(g) \rightarrow\left((\neg g) \rightarrow O\left(t^{\prime}\right)\right)$, so that in this case the contrary-to-duty obligation would be captured without use of the recension connective. This would be the case if we had $\models^{\mathcal{M}}(\neg g) \rightarrow O\left(t^{\prime}\right)$.

\subsection{Strategic reasoning.}

A particularly fruitful application of conditional logics has been to the study of strategic reasoning, particularly in the foundations of game theory. Extensive games involve a sequence of decisions made by players and a game state that unfolds during play. They are usually formalized as trees with vertices representing the state of play, and with edges representing moves of the game. Reasoning about such games often uses assertions of the form "player $i$ reasons that if the game were to reach a certain vertex (in a game tree), then some property, $\psi$, would hold". In particular, in extensive games the property $\psi$ here is often an assertion that subsequent play would further unfold in a certain way. The assertion $\psi$ is often tied to knowledge of the players about the decisions that would be made by other players at game vertices. A line of work has sought to explain game-theoretic solution concepts, such as backward induction, from epistemic foundations and formalizations of rationality involving maximization of payoffs under certain conditions, for example in works by Aumann [1], Halpern [11], Samet [25], and Stalnaker [27]. Conditional logics (with semantics on Ch-frames) have been used to formalize such forms of hypothetical reasoning in games, often in combination with epistemic logic or equivalent semantical formulations using operators on sets. As is shown below, this can be further augmented using the recension connective. It is also shown below that an extension of a standard framework for combining logical conditionals with epistemic operators allows the conditional to be that corresponding to a ternary relation semantics as in Section 8.

We assume familiarity with basic game-theoretic notions, but we now review some standard terminology for finite games. There is a set of players denoted $\{1, \ldots, N\}$. Each player, $i$, has a set of (pure) strategies $\Sigma_{i}$. If the game is in strategic (matrix) form, then these are atomic. The set of strategy profiles is $\Sigma=$ $\Sigma_{1} \times \ldots \times \Sigma_{N}$. Games are also often presented in extensive form using game trees. A game tree consists of a tree, with nodes labelled by the single player that moves from that node, and each edges from a node towards the leaves labelled by a possible move (actions) that the player can make at that point. A play consists of a path from the root of the tree to a leaf. Let $\Pi$ be the set of plays. Each player's set of nodes 
is partitioned into information sets, subject to some conditions for which the details are not required here. An information set is intended to describe a player's knowledge of the game node: if the game reaches an information set, the player at that set does not know which of the nodes in it has been reached. The partition is required to satisfy conditions so that each player knows its own past actions, but not necessarily those of other players. In the particular case of a game of perfect information, the game nodes all belong to singleton information sets. In an extensive form game, a strategy for player $i$ is a unique choice of action at each of its information sets. In an extensive form game, each player associates a real-valued payoff $u_{i}(p)$ with each play $p$. Each strategy profile in an extensive form game determines a play. In both extensive and strategic form games, each player $i$ has a payoff function $u_{i}: \Sigma \longrightarrow \mathbb{R}$. We let $u(\sigma)=\left(u_{1}(\sigma), \ldots, u_{N}(\sigma)\right)$ be the tuple of payoffs for all players for each strategy profile $\sigma$.

A number of approaches to epistemic game theory, including those mentioned above, use variants of a framework that we now describe. There is a set of worlds $W$. Each world determines how the game would proceed: concretely this is done by specifying a realization function $\rho: W \longrightarrow \Sigma$. Sometimes the realization determines the plays directly rather than via the set of strategies. The set $W$ carries additional structure in the form of a partition of its elements for each player. Often this is presented equivalently using the corresponding equivalence relation, or suitable unary operators on $\mathcal{P} W$ : in any case, this structure is sufficient to allow it to be used as a semantics of S5 epistemic logic in the standard way, as for example described in Priest [22]. Thus it gives a semantics to statements of knowledge of properties of worlds, and in particular these properties will often be tied to corresponding set of games under the realization. Moreover, this allows for theorems to be established regarding the relation between epistemic considerations (for example, "common knowledge of rationality") and game-theoretic notions such as solution concepts (for example, subgame perfect equilibria that can be found by backward induction); a key contribution is the work by Aumann [1] although this does not use an explicit formal logical language. We do not make use formally of the epistemic operators in the discussion below.

Example 5.3 (Recension in a centipede game.). Consider the extensive form game tree shown without payoffs in Figure 1. This tree represents a strategic situation with two players, labelled 1 and 2. Play starts

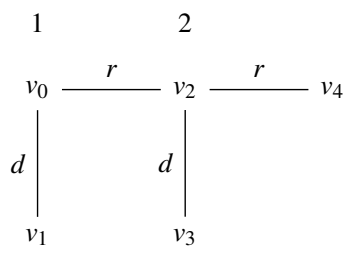

FIGURE 1. A Centipede Game Tree without Payoffs

at vertex (state) $v_{0}$. Let $u_{i}(v)$ be the payoff of each player $i$ at each terminal vertex $v$, and so also for the corresponding plays and strategy profiles. Let $u\left(v_{1}\right)=(1,0), u\left(v_{3}\right)=(0,2)$ and $u\left(v_{4}\right)=(2,1)$. Suppose also that 2 is rational in the sense that it chooses in such a way as to maximize its payoff. A standard (backward induction) argument would say that player 1 can reason (and so know) that if play reaches $v_{2}$, then player 1 will play d. Hence if player 1 is rational, in the sense of optimizing payoffs, and knows the same about player 2, she should choose $d$. Other rationality notions exist in the game theory literature.

There are four strategy profiles $s^{(1)}=(d, d), s^{(2)}=(d, r), s^{(3)}=(r, d), s^{(4)}=(r, r)$, where the left component of each pair shows the action chosen by player 1 , and the second component shows what player 2 would do if play reached $v_{2}$. Let $W=\left\{w_{1}, w_{2}, w_{3}, w_{4}\right\}$ where $\rho\left(w_{j}\right)=s^{(j)}$ for $j=1,2,3,4$.

Let $\psi$ be a propositional letter, valued so that

$$
v(\psi)=\left\{w \in W \mid v_{2} \text { lies on the play determined by } \rho(w)\right\}=\left\{w_{3}, w_{4}\right\} .
$$

Then, as a number of authors have argued, hypothetical reasoning of the form "if play reaches $v_{2}$, then $\theta$ " by player $i$, where the consequent $\theta$ is some assertion about how the game would unfold, can often be formalized using propositions of the form $\psi \rightarrow_{i} \theta$. In richer examples a more accurate description that 
reflects also the information available to players uses epistemic operators nested within this conditional, see Halpern [12]. Semantically, the Ch-frame transition function $f_{i}$, for each player $i$, carries the update of world that accompanies entertaining the idea that $v_{2}$ is reached. The form of this transition varies from example to example. In the Aumann analysis justifying backward induction, the world is unchanged under transition. In some other approaches the world is updated minimally to accommodate consistency with the hypothesis of a vertex being reached; such a transition can determine whether equilibria are supported by rational play that are not necessarily those found by backward induction, see Halpern [12] again, for example.

Implicit in the justification of backward induction is the ability to consider the combination

player 1 is rational and believes that player 2 is rational and yet, hypothetically, play reaches $v_{2}$.

In the case of the present example, player 1 is rational, while believing also that player 2 is rational, just when player 1 chooses $d$. This holds just in $w_{1}$ and $w_{2}$. Let $\phi$ be a letter valued so that $v(\phi)=\left\{w_{1}, w_{2}\right\}$.

Suppose that the transition function $f_{i}$ for player $i$ is such that $f_{i}\left(w_{1}, \llbracket \psi \rrbracket\right)=\left\{w_{3}\right\}, f_{i}\left(w_{2}, \llbracket \psi \rrbracket\right)=\left\{w_{4}\right\}$, $f_{i}\left(w_{j}, \llbracket \psi \rrbracket\right)=\left\{w_{j}\right\}$ for $j=3,4$, omitting the evident superscripts on the semantic interpretation brackets. We can thus write

$$
\models_{w_{j}} \phi \triangleright_{i} \psi \quad \text { and } \quad \models_{w_{k}} \neg\left(\phi \triangleright_{i} \psi\right)
$$

to capture satisfaction of this "and yet" hypothetical combination for $j=3,4$ and its negation for $k=1,2$. On the other-hand we have

$$
\vDash_{w_{3}} \theta \triangleright_{i} \psi \quad \text { and } \quad \vDash_{w_{4}} \neg\left(\theta \triangleright_{i} \psi\right)
$$

where $v(\theta)=\left\{w_{1}, w_{3}\right\}$, so that $\theta$ represents the rationality of player 2 in playing $d$.

The recension connective thus provides a method to describe hypothetical worlds (and so strategy profiles) in terms of the properties of the base world (and so strategy profile) from which the hypothesis was made. A similar kind of connection between worlds before and after a hypothetical move is also present in arguments by forward induction. In forward induction a player, say 2, considering possible play at some point, eliminates future possible actions by another player, say 1, based on a strong belief by 2 in the rationality of 1 at a previous move that takes play off the backward induction path. That is to say, if a player makes a move off the backward induction path, then (given belief in its rationality at that move) this was deliberate and transmits a signal about how it will play in the remaining game. Perea [21] describes forward induction in more detail.

The semantics of CCK on Ch-frames places no restrictions on the updating of worlds under any hypothesis, and no restriction on the relation between updates when the hypothesis is a combination of other hypotheses. This allows it to be used to formalize conditional statements in games, such as those discussed above. However, the Ch-frame transitions used in such examples are usually not representable in the sense of Section 8, except in quite trivial cases.

In many real games, for example chess, a player of the game cannot have knowledge or reason effectively about complete (total) plays of the game, or total strategies defined at all decision points, because of computational limitations. Rather, the player reasons about fragments of the game and tries to combine the results of such reasoning. Suppose that a world does not now determine a total play of the game. Define a partial strategy for a player to be a choice of action at each element of a subset of the information sets of that player. In other words, it is a partial (dependent) function from information sets to the available actions at each information set. Let $\widehat{\Sigma}_{i}$ be the set of partial strategies of player $i$. Let $\widehat{\Sigma}=\widehat{\Sigma}_{1} \times \ldots \times \widehat{\Sigma}_{N}$ be the set of partial-strategy profiles. Define a reification to be a map $\rho: W \longrightarrow \widehat{\Sigma}$. We use these ideas below to begin to develop aspects of game-theoretic reasoning using a semantics of the conditional on ternary relation frames. A fuller development would feature an integration of epistemic logic and semantics with such conditionals and semantics, and also formalization of game-theoretic rationality concepts suited to reification rather than realization. 
The reification notion allows us to define a ternary relation $R$ on the set of worlds by lifting given ternary relations $\widehat{R}$ on the set of partial strategies:

$$
R\left(w, w^{\prime}, w^{\prime \prime}\right) \quad \text { iff } \quad \widehat{R}\left(\rho(w), \rho\left(w^{\prime}\right), \rho\left(w^{\prime \prime}\right)\right)
$$

for all $w, w^{\prime}, w^{\prime \prime} \in W$. In particular, this applies where $\widehat{R}$ happens to be defined by a partial binary operation $\triangleright$ (a partial magma), so that for all partial strategies $\sigma, \sigma^{\prime}, \sigma^{\prime \prime}$ we have

$$
\widehat{R}\left(\sigma, \sigma^{\prime}, \sigma^{\prime \prime}\right) \quad \text { iff } \quad \sigma^{\prime \prime}=\sigma \triangleright \sigma^{\prime} \text { is defined. }
$$

If $\rho$ is an injective function, then $R$ will define a partial binary operation on $W$. There are a number of useful ternary relations, and partial magmas in particular, that allow one to compose strategy profiles (or plays). For example, one simple choice of partial magma on partial-strategy profiles is based on disjoint union, $\sqcup$ : for each player $i$, the partial strategy $\left(\sigma \sqcup \sigma^{\prime}\right)_{i}$ is defined when $\sigma_{i}$ and $\sigma_{i}^{\prime}$ are defined on disjoint subsets of $i$ 's game nodes; then $\sigma \sqcup \sigma^{\prime}$ is defined if $\left(\sigma \sqcup \sigma^{\prime}\right)_{i}$ is defined for each player $i$. A second interesting example is based on the (left to right) overwriting union, $\uplus$, on strategies. For each player $i$ and each node $x$ belonging to $i$, the partial strategy $\left(\sigma \uplus \sigma^{\prime}\right)_{i}$ at $x$ is: $\sigma_{i}^{\prime}(x)$ if $\sigma_{i}^{\prime}(x)$ is defined; $\sigma_{i}(x)$ if $\sigma_{i}^{\prime}(x)$ is not defined and $\sigma_{i}(x)$ is defined; undefined otherwise.

Let $x$ be a node of a game tree. The relation on worlds (defined by 9) allows us to consider a conditional in the usual way, that is, according to 12. Moreover, for any node $x$ of the game we can consider the set of worlds $U_{x}$ such that the reification of the world is a partial-strategy profile that defines an unbroken partial play that passes from the root and reaches $x$, but that reaches no other game nodes. Let $\phi_{x}$ be a propositional letter to be valued as $U_{x}$. We can then recover variants of statements of the form "if play reaches $x$, then ..." as propositions $\phi_{x} \rightarrow \psi$ for some $\psi$, where $\rightarrow$ agrees with $\rightarrow$ as in Section 8 . Similarly the connective $\triangleright$ agrees with $\triangleright$. With a semantics generated from a partial magma $\triangleright$ on partial strategies (lifted to worlds) in this way,

$$
\begin{aligned}
& \models_{w} \phi_{x} \rightarrow \psi \text { iff, for all } w^{\prime} \text { such that } w \triangleright w^{\prime} \text { is defined, if } \models_{w^{\prime}} \phi_{x} \text { then } \models_{w \triangleright w^{\prime}} \psi \\
& \vDash_{w} \psi \triangleright \phi_{x} \text { iff, there are } w^{\prime}, w^{\prime \prime} \text { with } w=w^{\prime} \triangleright w^{\prime \prime} \text { and } \models_{w^{\prime}} \psi \text { and } \vDash_{w^{\prime \prime}} \phi_{x} .
\end{aligned}
$$

In the particular case of the partial magma operation, $\sqcup$, based on disjoint union, the conditional $\phi_{x} \rightarrow \psi$ is true in worlds $w$ such that $\rho(w)$ considers only play not before node $x$ (apart from strategic choices incomparable with $x$ ), and so that any world $w^{\prime}$ with $\rho\left(w^{\prime}\right)$ disjoint from $\rho(w)$ giving play that reaches $x$ is such that the composite strategy $\rho\left(w \sqcup w^{\prime}\right)=\rho(w) \sqcup \rho\left(w^{\prime}\right)$ satisfies the condition $\psi$. The worlds $w \sqcup w^{\prime}$ here all determine plays through $x$. This particular choice thus gives a form of compositional reasoning in extensive form games. Different choices of partial magma give rise to different conditionals. The overwriting union, $\uplus$, for example, defines a transition $f$ in which $f\left(w, U_{x}\right)$ is a singleton that updates just those parts of $w$ so that the corresponding strategy matches $w$, except below $x$ where it matches the partial-strategy profile leading to $x$ determined by $U_{x}$. This is essentially the closest world updating used to formalize substantive rationality in many examples, see for example Halpern [12], but now dealing with worlds representing partial strategies as well as total ones. From an applied perspective, this process has the advantage that the semantics of the conditional is determined in a simple, coherent manner by lifting operations on the underlying game structure, rather than specifying a Ch-frame in some other, often more ad hoc, manner. This comes at the price of losing the full generality and flexibility of Ch-frames.

\section{Completeness and the Finite Model Property}

\subsection{Consistent Sets of Formulae.}

Let $L$ be any logic. A set $\Gamma$ of formulae is inconsistent over $L$ if $\Gamma \vdash_{L} \perp$, and consistent otherwise. As usual, consistency is equivalent to the existence of any formula $\phi$ such that $\Gamma \nvdash_{L} \phi$. A consistent set of formulae is maximal if it is not properly contained in any other consistent set. Let $\operatorname{MaxCon}(\mathrm{L})$ be the set of all maximal consistent sets of formulae (for $L$ ). For any formula $\phi$, let $|\phi|_{L} \subseteq \operatorname{Max} \operatorname{Con}(L)$ be the set of all maximal consistent sets containing $\phi$.

Proposition 6.1 collects together a number of standard facts about maximal consistent sets for boolean logics. An account of these properties for logics with modal operators appears in Lemmon [18] pp17-18.

\section{Proposition 6.1.}


(1) (Lindenbaum's Lemma) Every consistent set of formulae is contained in at least one maximal consistent set of formulae.

(2) $r_{\mathrm{L}} \phi$ if and only if $\phi \in \bigcap\{\beta \mid \beta \in \operatorname{Max} \operatorname{Con}(\mathrm{L})\}$.

(3) $\Gamma \vdash_{\mathrm{L}} \phi$ if and only if $\phi \in \bigcap\{\beta \mid \beta \in \operatorname{Max} \operatorname{Con}(\mathrm{L})$ and $\Gamma \subseteq \beta\}$.

(4) For every maximal consistent set $\beta$ and every formula $\phi$, either $\phi \in \beta$ or $\neg \phi \in \beta$.

(5) Every maximal consistent set is closed under derivability: if $\beta$ is a maximal consistent set, $\Gamma \subseteq \beta$ and $\Gamma \vdash_{\mathrm{L}} \phi$, then $\phi \in \beta$.

Proposition 6.2 collects together facts regarding maximal consistent sets for classes of conditional logics. It follows immediately from the closure of maximal consistent sets under derivability, and the particular axioms assumed. These facts are used in the completeness proofs below.

Proposition 6.2. Let $\mathrm{L}$ be a basic logic.

(1) Suppose that $\mathrm{L}$ contains $C R$. If $\beta$ is a maximal consistent set, $\phi \rightarrow \psi \in \beta$ and $\phi \rightarrow \theta \in \beta$, then $\phi \rightarrow(\psi \wedge \theta) \in \beta$.

(2) Suppose that $\mathrm{L}$ contains RCM. If $\beta$ is a maximal consistent set, $\phi \rightarrow \psi \in \beta$ and $\psi \rightarrow \theta \in \mathrm{L}$, then $\phi \rightarrow \theta \in \beta$.

Proof. For the first point, take $\Gamma=\{\phi \rightarrow \psi, \phi \rightarrow \theta\}$ and apply the final part of Proposition 6.1 with CR. For the second point, take $\Gamma=\{\phi \rightarrow \psi\}$ and apply the final part of Proposition 6.1 with RCM.

\subsection{Canonical Frames and Unwitnessed Adequacy.}

A Ch-frame $\mathcal{W}=\langle W, f\rangle$ is said to be canonic $^{4}$ if the set of worlds is the set of all L-maximal sets of sentences, $W=\operatorname{Max} \operatorname{Con}(\mathrm{L})$, and if $f$ is such that

$$
\phi \rightarrow \psi \in \beta \text { iff } f\left(\beta,|\phi|_{\mathrm{L}}\right) \subseteq|\psi|_{\mathrm{L}}
$$

for all $\beta, \phi, \psi$. It is said to be canonical if, additionally,

$$
\phi \triangleright \psi \in \beta \text { iff there is } \alpha \text { such that } \phi \in \alpha \text { and } \beta \in f\left(\alpha,|\psi|_{\mathrm{L}}\right)
$$

for all $\beta, \phi, \psi$. A valuation $v$ on a canonical frame such that $v(p)=|p|_{\mathrm{L}}$ for all $p \in$ Var is said to be canonical, in which case we have a canonical model.

There are well-defined operations on canonical frames that support the interpretation of a basal logic. The proof of Lemma 6.3 is a straightforward unwinding of the definitions.

Lemma 6.3. Let $\mathcal{W}=\langle W, f\rangle$ be a canonic frame. The operation $\rightarrow: \mathcal{P} W \times \mathcal{P} W \longrightarrow \mathcal{P} W$ satisfies $\mid \phi \rightarrow$ $\left.\psi\right|_{\mathrm{L}}=|\phi|_{\mathrm{L}} \rightarrow|\psi|_{\mathrm{L}}$ for all formulae $\phi, \psi$. If $\mathcal{W}$ is canonical, then the operation $\triangleright$ satisfies $|\phi \triangleright \psi|_{\mathrm{L}}=|\phi|_{\mathrm{L}} \triangleright|\psi|_{\mathrm{L}}$ for all formulae $\phi, \psi$.

Theorem 6.4 (Canonical Interpretation). Let $\mathcal{M}=(\mathcal{W}, v)$ be a canonical model. Then $\models_{\beta}^{\mathcal{M}} \phi$ iff $\phi \in \beta$ for every formula $\phi$ and every $\beta$ in $\mathcal{W}$. That is, $\llbracket \phi \rrbracket^{\mathcal{M}}=|\phi|_{\llcorner}$for every formula $\phi$.

Proof. This is a standard proof by induction on the structure of $\phi$, except for the cases for $\rightarrow$ and $\triangleright$. The treatment for the conditional $\rightarrow$ follows Theorem 2 in Chellas [4]. For the $\triangleright$-case,

$$
\begin{array}{rlrl} 
& \llbracket \phi \triangleright \psi \rrbracket^{\mathcal{M}} & \\
= & \llbracket \phi \rrbracket^{\mathcal{M}} \triangleright \llbracket \psi \rrbracket^{\mathcal{M}} & & \text { (by definition of the interpretation) } \\
= & |\phi|_{\mathrm{L}} \triangleright|\psi|_{\mathrm{L}} & & \text { (by the induction hypothesis) } \\
= & |\phi \triangleright \psi|_{\mathrm{L}} & & \text { (since } M \text { is canonical). }
\end{array}
$$

Corollary 6.5 (Unwitnessed Adequacy). If $\mathcal{M}$ is a canonical model for a logic $\mathrm{L}$, then

$$
\vdash_{\mathrm{L}} \phi \text { if } \vDash^{\mathcal{M}} \phi \text {. }
$$

Proof. By part 2 of Proposition 6.1, the theorems of $L$ are the propositional formulae that belong to every $L$-maximal set of formulae. These maximal sets are precisely the worlds in $\mathcal{M}$.

\footnotetext{
${ }^{4}$ These are the canonical standard frames of Chellas [4]
} 
An immediate consequence of Lemma 6.3, Theorem 6.4 and Corollary 6.5 is that a conditional logic with recension and a canonical model must be basal. A meaningful completeness result will be established provided it can be shown that a canonical model exists. Such a model is constructed below.

\subsection{Functional Frames.}

A logic is said to be serial if it includes

$(\mathrm{D} \rightarrow)$

$$
\neg(\psi \rightarrow \perp)
$$

for all formulae $\psi$. A frame $\langle W, f\rangle$ is said to be serial if $f(w, \psi) \neq \emptyset$ for all $w \in W$. In this section, attention is restricted to serial logics and frames. In particular, this excludes frames satisfying the full identity principle, $\phi \rightarrow \phi$ for all $\phi$, and that simultaneously interpret $\perp$ as $\emptyset$. Stalnaker [28] avoids this using an absurd world in each frame.

A logic is partial functional if it includes

$\left(\mathrm{G}^{1,0,1,0 \rightarrow)}\right.$

$$
(\psi \rightarrow \theta) \vee(\psi \rightarrow \neg \theta)
$$

for all $\psi, \theta$. A frame $\langle W, f\rangle$ is partial functional if for all $w$ and all $\psi$, there is some $w^{\prime}$ such that $f(w, \llbracket \psi \rrbracket) \subseteq$ $\left\{w^{\prime}\right\}$. A logic is functional if it is serial and partially functional. Similarly, frames are functional if they are serial and partially functional. The frames considered by Stalnaker [28] and many others are functional. Proposition 6.6 below is immediately verified.

Proposition 6.6. If a frame is serial, then $\mathrm{D} \rightarrow$ is valid on it. If a frame is partially functional, then $\mathrm{G}^{1,0,1,0} \rightarrow$ is valid on it.

Lemma 6.7. Let $\phi$ and $\psi$ be formulae of the basal and partial functional logic L. Suppose that $\phi \triangleright \psi \in \beta$ for some maximal consistent $\beta$. Define the set of formulae $\Delta_{\psi}=\{\psi \rightarrow \theta \mid \theta \in \beta\}$. Then the set of formulae $\Gamma_{\phi, \psi}=\{\phi\} \cup \Delta_{\psi}$ is consistent.

Proof. Suppose that $\Gamma_{\phi, \psi}$ is inconsistent. There are then $\phi_{1}, \ldots, \phi_{n} \in \Gamma_{\phi, \psi}$ such that $\vdash_{\mathrm{L}} \phi_{1} \wedge \ldots \wedge \phi_{n} \rightarrow \perp$. Given the idempotence of conjunction and the normality of $\rightarrow$, the set $\Delta_{\psi}$ is consistent. The only interesting case is of the form $\vdash_{\mathrm{L}}(\phi \wedge(\psi \rightarrow \theta)) \rightarrow \perp$ where $\theta \in \beta$. In this case $\vdash_{\mathrm{L}} \phi \rightarrow \neg(\psi \rightarrow \theta)$. Partial functionality gives $\vdash_{\mathrm{L}}(\psi \rightarrow \theta) \vee(\psi \rightarrow \neg \theta)$, and from this it follows that $\vdash_{\mathrm{L}}(\neg(\psi \rightarrow \theta)) \rightarrow(\psi \rightarrow \neg \theta)$. Therefore $\vdash_{\mathrm{L}} \phi \rightarrow(\psi \rightarrow \neg \theta)$. By RCCADJU, it follows that $\vdash_{\mathrm{L}}(\phi \triangleright \psi) \rightarrow \neg \theta$. Since maximal consistent sets are closed under derivability, $\neg \theta \in \beta$, contradicting $\theta \in \beta$.

Theorem 6.8 (Existence of Functional Canonical Models). Let $\mathrm{L}$ be a basal and functional conditional logic with recension. Let $W$ be the set of maximal consistent sets of formulae, MaxCon(L). Let $W=\langle W, f\rangle$ be a Ch-frame such that

$$
\beta \in f\left(\alpha,|\psi|_{\mathrm{L}}\right) \quad \text { iff } \quad\{\theta \in \text { Form } \mid \psi \rightarrow \theta \in \alpha\} \subseteq \beta .
$$

for all $\alpha, \beta \in W$ and formulae $\psi$. Then the structure is a canonical frame for $L$.

Proof. It is easily verified that the frame is functional. Chellas [4] shows that the structure is a canonic frame for a normal conditional logic. Thus it remains to check the condition for recension in the definition of canonical frame.

Suppose that $\beta \in|\phi|_{\mathrm{L}} \triangleright|\psi|_{\mathrm{L}}$. By definition there is $\alpha \in|\phi|_{\mathrm{L}}$ such that $\beta \in f\left(\alpha,|\psi|_{\mathrm{L}}\right)$. Now $\vdash_{\mathrm{L}}(\phi \triangleright \psi) \rightarrow$ $(\phi \triangleright \psi)$, so $\vdash_{\mathrm{L}} \phi \rightarrow(\psi \rightarrow(\phi \triangleright \psi))$ by RCCADJC. Since $\phi \in \alpha$ it follows that $\psi \rightarrow(\phi \triangleright \psi) \in \alpha$, because maximal consistent sets are closed under derivability. By construction $\beta \in f\left(\alpha,|\psi|_{\mathrm{L}}\right)$ then gives $\phi \triangleright \psi \in \beta$, from which $\beta \in|\phi \triangleright \psi|_{\mathrm{L}}$ by definition. Therefore $|\phi|_{\mathrm{L}} \triangleright|\psi|_{\mathrm{L}} \subseteq|\phi \triangleright \psi|_{\mathrm{L}}$.

Suppose $\beta \in|\phi \triangleright \psi|_{\mathrm{L}}$, so $\phi \triangleright \psi \in \beta$. Define $\Gamma_{\phi, \psi}=\{\phi\} \cup\{\psi \rightarrow \theta \mid \theta \in \beta\}$. Using partial functionality, by Lemma 6.7 the set $\Gamma_{\phi, \psi}$ is consistent. By part 1 of Proposition 6.1, the set $\Gamma_{\phi, \psi}$ is contained in some maximal consistent set $\alpha$. Since $\phi \in \alpha$ it follows, by definition, that $\alpha \in|\phi|_{\mathcal{L}}$.

Suppose, for a contradiction, that $\beta \notin|\phi|_{\mathrm{L}} \triangleright|\psi|_{\mathrm{L}}$. Then, by definition of the operation $\triangleright$, it must be that $\beta \notin f\left(\alpha^{\prime},|\psi|_{\mathrm{L}}\right)$ for all $\alpha^{\prime}$ such that $\alpha^{\prime} \in|\phi|_{\mathrm{L}}$. In particular, it must be that $\beta \notin f\left(\alpha,|\psi|_{\mathrm{L}}\right)$. It must then be that there is some $\theta \notin \beta$ with $\psi \rightarrow \theta \in \alpha$. Since $\beta$ is maximal, $\neg \theta \in \beta$. Then $\psi \rightarrow \neg \theta \in \alpha$, since $\psi \rightarrow \neg \theta \in \Gamma_{\phi, \psi} \subseteq \alpha$.

By Proposition 6.2 part 1, $\psi \rightarrow(\theta \wedge \neg \theta) \in \alpha$. By Proposition 6.2 part 2, $\psi \rightarrow \perp \in \alpha$. However, since $\mathrm{L}$ is serial $\neg(\psi \rightarrow \perp) \in \alpha$, giving a contradiction. Therefore the inclusion $|\phi \triangleright \psi|_{\mathrm{L}} \subseteq|\phi|_{\mathrm{L}} \triangleright|\psi|_{\mathrm{L}}$ holds. 
The following corollary follows immediately from Theorem 6.8 since it provides the witness canonical model to accompany Corollary 6.5 .

Corollary 6.9 (Completeness of Functional Logics on Functional Frames). Let $\mathrm{L}$ be a basal, functional logic, and let $\mathcal{M}$ be a canonical model as in Theorem 6.8. Then

$$
\vdash_{\llcorner} \phi \text { iff } \vDash^{\mathcal{M}} \phi
$$

for all formulae $\phi$.

The recension connective imposes a condition on frames demanding that certain worlds are related when a recension formula holds at certain worlds. Proposition 6.10 shows that this condition agrees with condition 10 in Theorem 6.8 demanded on the relation by the conditional connective. In other words, the relation constructed in the standard (modal) way from the $\rightarrow$ connective (regarded as a necessity operator) has an inverse that agrees with the relation constructed from the $\triangleright$ operator (regarded, essentially, as a possibility operator).

Proposition 6.10. Let $\mathrm{L}$ be a basal logic. Then

$$
\{\phi \triangleright \psi \mid \phi \in \alpha\} \subseteq \beta \quad \text { iff } \quad\{\theta \mid \psi \rightarrow \theta \in \alpha\} \subseteq \beta
$$

for all $\beta \in \operatorname{Max} \operatorname{Con}(L)$ and $\psi \in$ Form.

Proof. The RCCADJ rules yield $\vdash_{L}((\psi \rightarrow \theta) \triangleright \psi) \rightarrow \theta$ and $\vdash_{L} \phi \rightarrow(\psi \rightarrow(\phi \triangleright \psi))$. The result then follows immediately from the closure of maximal consistent sets under derivability.

\subsection{Completeness without Functionality.}

It was noted in Section 4 that $\mathrm{Ch}$-frames induce a CMB-algebra and in Section 3 that all CMB-algebras are in fact double-normal. This allows us to regard both the conditional and the recension to be regarded as normal modal operators. As usual, in terms of frames, the conditional is the family of necessity operators for the family of relations corresponding to the transition. The recension is simply the family of possibility operators corresponding to the inverses of those relations. This gives a way to show completeness without the functionality assumptions of Subsection 6.2, by generalizing the canonical model existence proof of Theorem 6.8.

Theorem 6.11 (Existence of Canonical Models). Let $\mathrm{L}$ be a basal conditional logic with recension. Then the construction of Theorem 6.8 defines a canonical model.

Proof. The only part of the proof for which functionality was used previously was in showing that $|\phi \triangleright \psi|_{\mathrm{L}} \subseteq$ $|\phi|_{\llcorner} \triangleright|\psi|_{\mathrm{L}}$. Let $\beta \in|\phi \triangleright \psi|_{\mathrm{L}}$. Define $\Gamma=\{\phi\} \cup\{\neg \theta \mid \neg(\theta \triangleright \phi) \in \beta\}$. The consistency of this is verified in Lemma 6.12. Since $\Gamma$ is consistent, it has a maximal consistent extension, say $\alpha$. Now $\{\theta \triangleright \psi \mid \theta \in \alpha\} \subseteq \beta$ by construction of $\alpha$ from $\Gamma$. By Proposition 6.10 the condition $\{\theta \mid \psi \rightarrow \theta \in \alpha\} \subseteq \beta$ holds, and hence $\beta \in f\left(\alpha,|\psi|_{\mathrm{L}}\right)$. Since $\alpha \in|\phi|_{\mathrm{L}}$, it follows that $\beta \in|\phi|_{\mathrm{L}} \triangleright|\psi|_{\mathrm{L}}$.

Lemma 6.12. The set $\Gamma$ appearing in the proof of Theorem 6.11 is consistent.

Proof. The proof is of the completely standard kind using normality. Suppose that $\vdash_{L}\left(\phi_{1} \wedge \ldots \phi_{n}\right) \rightarrow \perp$. There are two cases, depending on whether or not one of the $\phi_{i}$ is identical to $\phi$. Attention can be restricted to the case $n=2$ without loss of generality. Let $\Delta=\{\neg \theta \mid \neg(\theta \triangleright \phi) \in \beta\}$.

For the first case, suppose that $\phi_{1}, \phi_{2} \in \Delta$. For $i=1,2$, let $\phi_{i}$ be $\neg \theta_{i}$ where $\neg\left(\theta_{i} \triangleright \phi\right) \in \beta$. By assumption, $\vdash\left\llcorner\theta_{1} \vee \theta_{1}\right.$, and so $\vdash \mathrm{L} \phi \rightarrow\left(\theta_{1} \vee \theta_{1}\right)$. From RCCML it can be inferred that $\vdash_{L}(\phi \triangleright \psi) \rightarrow\left(\left(\theta_{1} \vee \theta_{2}\right) \triangleright \psi\right)$. Now $\neg\left(\theta_{1} \triangleright \psi\right) \wedge \neg\left(\theta_{2} \triangleright \psi\right) \in \beta$. Therefore $\neg\left(\left(\theta_{1} \triangleright \psi\right) \vee\left(\theta_{2} \triangleright \psi\right)\right) \in \beta$, and $\left(\left(\theta_{1} \triangleright \psi\right) \vee\left(\theta_{2} \triangleright \psi\right)\right) \notin \beta$. Therefore $\left(\left(\theta_{1} \vee \theta_{2}\right) \triangleright \psi\right) \notin \beta$ by CCR. But then $\phi \triangleright \psi \notin \beta$ by closure under derivability. This contradiction shows that $\Delta$ cannot be inconsistent.

For the second case, suppose that $\phi_{1}=\phi \notin \Delta$ and $\phi_{2}=\neg \theta$ where $\neg(\theta \triangleright \phi) \in \beta$. By assumption, $\vdash \mathrm{L}(\phi \wedge \neg \theta) \rightarrow \perp$, so $\vdash \mathrm{L} \phi \rightarrow \theta$. By RCCML, $\vdash \mathrm{L}(\phi \triangleright \psi) \rightarrow(\theta \triangleright \psi)$. Now $\theta \triangleright \psi \notin \beta$, and so $\phi \triangleright \psi \notin \beta$ and $\phi \in \Delta$, giving the required contradiction.

\subsection{Filtrations, Finite Models and Decidability.}


Let $\mathcal{M}$ be a model based on a frame with carrier set $W$. Let $\Gamma$ be a set of formulae that is closed under subformulae. Define an equivalence relation $\equiv$ on $W$ by

$$
w \equiv w^{\prime} \quad \text { iff, } \quad \models_{w}^{\mathcal{M}} \phi \text { iff } \models_{w^{\prime}}^{\mathcal{M}} \phi \text { for all } \phi \in \Gamma,
$$

for all $w, w^{\prime}$ in $W$. Let $[w]^{\Gamma}$ be the equivalence class of each $w \in W$. For any $X \subseteq W$, let $[X]^{\Gamma}=\left\{[w]^{\Gamma} \mid w \in\right.$ $X\}$ be the set of equivalence classes of members of $X$. We omit the superscript on equivalence classes and sets of equivalence classes when the set of formulae is evident from the context.

Let $\mathcal{M}=\langle\mathcal{W}, v\rangle$ be a model, with $\mathcal{W}=\langle W, f\rangle$ the underlying frame. Let $\Gamma$ be a set of formulae that is closed under subformulae. A filtration of $\mathcal{M}$ through $\Gamma$ is a model $\mathcal{M}^{*}=\left\langle\mathcal{W}^{*}, v^{*}\right\rangle$ based on a frame $W^{*}=\left\langle W^{*}, f^{*}\right\rangle$ such that $W^{*}=[W], v^{*}(\phi)=[v(\phi)] \subseteq W^{*}$ for all propositional variables $\phi$, and so that the following three conditions hold:

(A) $[f(w, X)] \subseteq f^{*}([w],[X])$

(B) If $\left[w^{\prime}\right] \in f^{*}([w],[X]), \phi \rightarrow \psi \in \Gamma, X=\llbracket \phi \rrbracket^{\mathcal{M}}$ and $\models_{w}^{\mathcal{M}} \phi \rightarrow \psi$, then $\models_{w^{\prime}}^{\mathcal{M}} \psi$

(C) If $\left[w^{\prime}\right] \in f^{*}([w],[X]), \phi \triangleright \psi \in \Gamma, X=\llbracket \psi \rrbracket^{\mathcal{M}}$ and $\models_{w}^{\mathcal{M}} \phi$, then $\models_{w^{\prime}}^{\mathcal{M}} \phi \triangleright \psi$.

There is always one filtration (the minimal filtration) defined by taking equality in condition A.

The standard modal technique of filtrations transfers to the treatment of the conditional connective as a family of necessity operators, see Chellas [4]. This uses the conditions A and B. This is further extended to the recension connective, treating it as a family of possibility operators, by adding the condition $\mathrm{C}$.

Theorem 6.13. Let $\Gamma$ be a set of formulae that is closed under subformulae. Let $\mathcal{M}^{*}$ be a filtration of a model through $\Gamma$. Then for every $\phi \in \Gamma$ and every $w$ in $\mathcal{M}$

$$
\models_{w}^{\mathcal{M}} \phi \quad \text { iff } \quad \models_{[w]}^{\mathcal{M}^{*}} \phi
$$

Moreover,

$$
\models^{\mathcal{M}} \phi \quad \text { iff } \quad \models^{\mathcal{M}^{*}} \phi .
$$

Proof. The proof is standard, and goes by induction on the structure of the formula $\phi$. Condition B is used in the case for the conditional connective. In the case where the formula takes the form $\phi \triangleright \psi$, it is required to show that

$$
w^{\prime} \in f\left(\llbracket \phi \rrbracket^{\mathcal{M}}, \llbracket \psi \rrbracket^{\mathcal{M}}\right) \quad \text { iff } \quad\left[w^{\prime}\right] \in f^{*}\left(\left[\llbracket \phi \rrbracket^{\mathcal{M}}\right],\left[\llbracket \psi \rrbracket^{\mathcal{M}}\right]\right) .
$$

Sufficiency (from left to right) follows using condition A. Necessity (from right to left) follows directly using the induction hypothesis and condition $\mathrm{C}$.

The canonical model construction of Subsection 6.4, together with the soundness and (unwitnessed) adequacy results shows that CCK is determined by the class of models on Ch-frames. The filtration technique shows that in fact there is always a finite model to determine each sentence. For any formula $\phi$, let $\Gamma_{\phi}$ be the set of subformulae of $\phi$. If $\phi$ is derivable, then it will be valid in a canonical model as in Theorem 6.11. A filtration, $\mathcal{M}_{\phi}$, of this through $\Gamma_{\phi}$ is then finite and satisfies $\phi$. Decidability of $\phi$ follows immediately by checking validity in $\mathcal{M}_{\phi}$.

Theorem 6.14. For each propositional formula $\phi$, there is a finite model $\mathcal{M}$ such that

$$
\text { rcck } \phi \text { iff } \models^{\mathcal{M}} \phi \text {. }
$$

Corollary 6.15. CCK is decidable.

Segerberg [26] studies extensions of CK. In particular, he states a sequence of additional axioms that together give the well-known logic VC of Lewis [19]. Segerberg gives corresponding frame conditions characterizing each of these axioms. One would wish to produce finite models for these extensions and the corresponding frames. The filtration technique works directly for all of these axioms VC except that labelled \#7 by Segerberg, which says $\phi \rightarrow \psi, \phi \rightarrow \theta \vdash \mathrm{vc}(\phi \wedge \psi) \rightarrow \theta$. The corresponding frame condition is that labelled c7, which is $f(w, S) \cap T \neq \emptyset$ implies $f(w, S \cap T) \subseteq f(w, S)$ for all $w, S, T$. The key step for each axiom extension is to show that the class of corresponding frames is closed under application of the minimal filtration. This is not the case for \#7 and c7. Segerberg relates a generalization of the notion of filtration, the Strevens filtration, similarly yielding finite models. For filtrations on a canonical model, via a finite set of sentences $\Gamma$, a corresponding filtration theorem holds (analagous to Theorem 6.13 in this 
paper). Segerberg gives a particular Strevens filtration inspired by the minimal filtration. He then shows that each of the classes of frames, including those characterized by $\mathrm{c} 7$, are closed under application of that particular Strevens filtration. Thus a finite model for VC is constructed. This Strevens filtration technique continues to work if we add the recension connective to the language. The only point that requires checking is that the induction step of the modified filtration theorem goes through, and indeed it it is almost trivial to verify that the Strevens filtration condition ((iii') in Segerberg [26]) is sufficient to give this result.

\section{Minimal Models}

Scott-Montague minimal models can be used to provide a semantics for non-normal modal logics. Recall that, for a single modal necessity operator, $\square$, the semantics attaches to each world, $w$, the set of formulae $\phi$ for which $\square \phi$ should hold at $w$. This can be achieved using frames consisting of a set of worlds, $W$, and a function $F: W \longrightarrow \mathcal{P P} W$. Minimal models can also be used to provide a semantics for conditional logics without normality conditions, since the conditional is treated as a family of necessity operators indexed by the interpretations of the possible hypotheses of each conditional. The results above indicate that the RCCADJ rules lead to the normality of the conditional and recension connectives when viewed as modal connectives. The use of minimal models below sheds further light on the fact that the RCCADJ rules lead to normality and a relational representation of models. The terminology of this section follows Chellas $[4,5]$. We write the intersection of a family, $\mathcal{F}$, of sets in the (unindexed) form $\cap \mathcal{F}$, meaning $\bigcap_{X \in \mathcal{F}} X$.

A minimal frame is a pair $\mathcal{W}=\langle W, F\rangle$ where $F: W \times \mathcal{P} W \longrightarrow \mathcal{P} \mathcal{P} W$. In a minimal model of conditional logic, $\mathcal{M}$, propositional variables are valued in the same way as on $\mathrm{Ch}$-frames, and all clauses of the interpretation $\llbracket-\rrbracket^{\mathcal{M}}: \mathcal{L}(\rightarrow, \triangleright) \longrightarrow \mathcal{P} W$ are the same except that

$$
w \in \llbracket \phi \rightarrow \psi \rrbracket^{\mathcal{M}} \quad \text { iff } \quad \llbracket \psi \rrbracket^{\mathcal{M}} \in F\left(w, \llbracket \phi \rrbracket^{\mathcal{M}}\right)
$$

for all $w, \phi, \psi$.

A minimal frame is supplemented if $F(w, X)$ is an upper set in the inclusion order on $\mathcal{P} W$ for all $w \in W$ and $X \subseteq W$ : that is,

$$
\text { if } Y \in F(w, X) \text { and } Y \subseteq Z \text { then } Z \in F(w, X)
$$

for all $Y, Z \subseteq W$. A minimal frame is augmented if it is supplemented and each $F(w, X)$ contains a least element. The least element, if it exists, must be $\cap F(w, X)$. Minimal models are described as supplemented or augmented if their underlying minimal frames are, respectively, supplemented or augmented.

An augmented minimal frame $\langle W, F\rangle$ can be constructed from a Ch-frame $\langle W, f\rangle$ by defining

$$
Y \in F(w, X) \text { iff } f(w, X) \subseteq Y
$$

for all $w \in W$ and $X, Y \subseteq W$. A Ch-frame $\langle W, f\rangle$ can be constructed from an augmented minimal frame $\langle W, F\rangle$ by

$$
w^{\prime} \in f(w, X) \text { iff } w^{\prime} \in \bigcap F(w, X)
$$

for all $w, X$. This gives a bijection between augmented minimal frames and Ch-frames. Moreover, any Ch-frame model $\mathcal{M}$ is pointwise equivalent to the corresponding augmented minimal model $\mathcal{M}^{\prime}$, in the sense that $\llbracket \phi \rrbracket^{\mathcal{M}}=\llbracket \phi \rrbracket^{\mathcal{M}^{\prime}}$ for all $\phi$.

Soundness of RCCADJ in a minimal model $\mathcal{M}^{\prime}=\langle W, F\rangle$ requires

$$
\llbracket \phi \triangleright \psi \rrbracket^{\mathcal{M}^{\prime}} \subseteq \llbracket \theta \rrbracket^{\mathcal{M}^{\prime}} \quad \text { iff } \quad \llbracket \phi \rrbracket^{\mathcal{M}^{\prime}} \subseteq \llbracket \psi \rightarrow \theta \rrbracket^{\mathcal{M}^{\prime}}
$$

for any $\phi, \psi, \theta$. Combining this with the interpretation of the conditional connective gives

$$
\llbracket \phi \triangleright \psi \rrbracket^{\mathcal{M}^{\prime}} \subseteq \llbracket \theta \rrbracket^{\mathcal{M}^{\prime}} \quad \text { iff } \quad \llbracket \theta \rrbracket^{\mathcal{M}^{\prime}} \in \bigcap_{w \in \llbracket \phi \rrbracket^{\mathcal{M}^{\prime}}} F\left(w, \llbracket \psi \rrbracket^{\mathcal{M}^{\prime}}\right) .
$$

Now consider what is required for RCCADJ to be sound with all valuations on a minimal frame. Since $\theta$ may be a propositional letter with any valuation, the set $\bigcap_{w \in \llbracket \phi \rrbracket^{\mathcal{M}^{\prime}}} F\left(w, \llbracket \psi \rrbracket^{\mathcal{M}^{\prime}}\right)$ is an upper set in $\mathcal{P} W$ and that $\llbracket \phi \triangleright \psi \rrbracket^{\mathcal{M}^{\prime}}$ is the smallest element. Moreover, for any $w \in W$ and $Y \subseteq W$ we can consider a valuation of $\phi, \psi \in \operatorname{Var}$ such that $\llbracket \phi \rrbracket^{\mathcal{M}^{\prime}}=\{w\}$ and $\llbracket \psi \rrbracket^{\mathcal{M}^{\prime}}=Y$. Hence, the minimal frame must be augmented. Let 
the corresponding model on a Ch-frame have transition $f$. Since $f(w, Y)$ is the smallest element of each $F(w, Y)$ for any $w, Y$, we have that $\bigcup_{w \in X} f(w, Y)$ is the smallest element of $\bigcap_{w \in X} F(w, Y)$. We thus have

$$
\llbracket \theta \rrbracket^{\mathcal{M}^{\prime}} \in \bigcap_{w \in \llbracket \phi \rrbracket^{\mathcal{M}^{\prime}}} F\left(w, \llbracket \psi \rrbracket^{\mathcal{M}^{\prime}}\right) \quad \text { iff } \quad f\left(\llbracket \phi \rrbracket^{\mathcal{M}}, \llbracket \psi \rrbracket^{\mathcal{M}}\right) \subseteq \llbracket \theta \rrbracket^{\mathcal{M}}
$$

and so

$$
\llbracket \phi \triangleright \psi \rrbracket^{\mathcal{M}^{\prime}}=\llbracket \phi \triangleright \psi \rrbracket^{\mathcal{M}}
$$

where $\mathcal{M}$ is the Ch-frame model on $\langle W, f\rangle$ using the same valuation as $\mathcal{M}^{\prime}$. This result is summarized in an algebraic form in Proposition 7.1.

Proposition 7.1. Let $\langle W, F\rangle$ be a minimal frame. Suppose that $\mathcal{P} W$ carries the structure of a CMB-algebra, with the operation $\rightarrow$ satisfying

$$
w \in Y \rightarrow Z \quad \text { iff } Z \in F(w, Y)
$$

for all $w \in W$ and $Y, Z \in \mathcal{P} W$. Then the frame is augmented, and

$$
X \triangleright Y=\bigcap\left(\bigcap_{w \in X} F(w, Y)\right)
$$

for all $X, Y \in \mathcal{P} W$. The corresponding Ch-frame $\langle W, f\rangle$ has has $X \triangleright Y=f(X, Y)$ and $X \rightarrow Y=\{w \in W \mid$ $f(w, X) \subseteq Y\}$ for all $X, Y$ for the same $C M B$-algebra structure.

Proof. Using the relationship 7 we have that $X \triangleright Y \subseteq Z$ iff $Z \in \bigcap_{w \in X} F(w, Y)$ for all $X, Y \in \mathcal{P} W$. Therefore $\bigcap_{w \in X} F(w, Y)$ is an upper set in $\mathcal{P} W$ with least element $X \triangleright Y$. Since this holds for arbitrary $X, Y$, it follows that $\langle W, F\rangle$ is augmented, and so $f(w, Y)$ is the minimal element of $F(w, Y)$ for all $w, Y$. Therefore $\bigcup_{w \in X} f(w, Y)$ is the minimal element of $\bigcap_{w \in X} F(w, Y)$, and hence $X \triangleright Y=f(X, Y)$.

\section{Ternary Relation Semantics}

The frame semantics of the conditional and recension connectives of Section 4 treats them in the same manner as families of unary normal modal operators, with the indexing over the family of propositions, and using similarly indexed families of binary relations on the frame side. As shown above, this induces the structure of a CMB-algebra on the powerset of the carrier of a frame. An alternative way to induce such structure is to start with a single ternary relation on frames.

Define $\langle W, R\rangle$ to be a ternary relation frame where $W$ is the carrier set and $R \subseteq W \times W \times W$. There are binary operations $>$ and $\rightarrow$ on $\mathcal{P} W$ given by

$$
\begin{aligned}
& w \in U \triangleright U^{\prime} \quad \text { iff } \quad \text { there exist } w^{\prime \prime} \in U \text { and } w^{\prime} \in U^{\prime} \text { such that } R\left(w^{\prime}, w^{\prime \prime}, w\right) \\
& w \in U \rightarrow U^{\prime} \quad \text { iff } \quad \text { for all } w^{\prime}, w^{\prime \prime}, \text { if } w^{\prime} \in U \text { and } R\left(w, w^{\prime}, w^{\prime \prime}\right) \text { then } w^{\prime \prime} \in U^{\prime}
\end{aligned}
$$

for all $w \in W$ and $U, U^{\prime} \subseteq W$. The point of these definitions is to give the adjoint relationship

$$
U \triangleright U^{\prime} \subseteq U^{\prime \prime} \quad \text { iff } \quad U \subseteq U^{\prime} \rightarrow U^{\prime \prime}
$$

for all $U, U^{\prime}, U^{\prime \prime} \subseteq W$, which should be compared to 7. A ternary relation frame therefore induces the structure of a CMB-algebra on $\mathcal{P} W$.

A number of well-known logics have an adjoint relationship, similar to RCCADJ, in the form

$$
\frac{(\phi>\psi) \rightarrow \theta}{\overline{\phi \rightarrow(\psi \rightarrow \theta)}}
$$

linking a substructural or intensional conjunction-like connective, written here $\boldsymbol{\triangleright}$, to a form of implication, $\rightarrow$. This includes various members of the families of relevant logics, linear logics, and bunched logics: see Priest [22] and Pym [23], for example. Several of these have a semantics that uses a ternary relation frame and an interpretation $\llbracket \phi \rrbracket^{\mathcal{M}} \in \mathcal{P} W$ of formulae $\phi$ on a model $\mathcal{M}$ on a ternary frame $\langle W, R\rangle$ with

$$
\begin{aligned}
& \llbracket \phi>\psi \rrbracket^{\mathcal{M}}=\llbracket \phi \rrbracket^{\mathcal{M}}-\llbracket \psi \rrbracket^{\mathcal{M}} \\
& \llbracket \phi \rightarrow \psi \rrbracket^{\mathcal{M}}=\llbracket \phi \rrbracket^{\mathcal{M}} \rightarrow \llbracket \psi \rrbracket^{\mathcal{M}} .
\end{aligned}
$$

In famous special cases the connectives and operations are commutative and associative, and in many cases the relation is restricted to be the graph of a binary, partial monoidal operation on $W$. 
A Ch-frame $\langle W, f\rangle$ is representable when

$$
w^{\prime \prime} \in f\left(w, U^{\prime}\right) \quad \text { iff } \quad w^{\prime \prime} \in f\left(w,\left\{w^{\prime}\right\}\right) \text { for some } w^{\prime} \in U^{\prime}
$$

for all $w, w^{\prime \prime} \in W$ and $U^{\prime} \subseteq W$. The distributivity condition,

$$
f\left(w, \bigcup_{i \in I} U_{i}\right)=\bigcup_{i \in I} f\left(w, U_{i}\right)
$$

for all $w \in W$ and families ( $U_{i} \subseteq W \mid i \in I$ ), is equivalent to the representability condition. In particular $f(w, \emptyset)=\emptyset$. Serial and functional frames are therefore not representable. Proposition 8.1 shows that representable $\mathrm{Ch}$-frames are equivalent to ternary relation frames. The proof is a simple verification. The fact that Ch-frames can be represented as ternary frames whilst translating the conditional connective $\rightarrow$ into substructural $\rightarrow$ was discussed by Beall et al [2] using the distributivity condition.

Proposition 8.1. There is a bijective correspondence between representable Ch-frames $\langle W, f\rangle$ and ternary relation frames $\langle W, R\rangle$ via

$$
w^{\prime \prime} \in f\left(w,\left\{w^{\prime}\right\}\right) \quad \text { iff } \quad R\left(w, w^{\prime}, w^{\prime \prime}\right),
$$

for all $w, w^{\prime}, w^{\prime \prime} \in W$. This bijection moreover makes the operators $\triangleright$ and $\triangleright$ on $\mathcal{P} W$ equal; the operations $\rightarrow$ and $\rightarrow$ are also equal to each other.

The consequence of Proposition 8.1 is that the interpretation on a ternary frame of a language with just the connectives $>$ and $\rightarrow$ added to the usual boolean connectives matches the interpretation of the basic language $\mathcal{L}(\triangleright, \rightarrow)$ on Ch-frames. In particular, $\llbracket \phi \triangleright \psi \rrbracket^{\mathcal{M}}=\llbracket \phi \triangleright \psi \rrbracket^{\mathcal{M}^{\prime}}$ and $\llbracket \phi \rightarrow \psi \rrbracket^{\mathcal{M}}=\llbracket \phi \rightarrow \psi \rrbracket^{\mathcal{M}^{\prime}}$ for all $\phi, \psi$, where $\mathcal{M}$ and $\mathcal{M}^{\prime}$ are, respectively, the models with the same valuation on the ternary relation frame and the Ch-frame.

It is known that the Ch-frames satisfying the finite distributivity conditions, $f(w, \emptyset)=\emptyset$ and $f\left(w, U_{1} \cup\right.$ $\left.U_{2}\right)=f\left(w, U_{1}\right) \cup f\left(w, U_{2}\right)$ for all $w, U_{1}, U_{2}$, are determined (in the sense of giving soundness and completeness) by adding the axioms

$$
\perp \rightarrow \perp \quad \text { and } \quad\left(\left(\phi_{1} \rightarrow \psi\right) \wedge\left(\phi_{2} \rightarrow \psi\right)\right) \leftrightarrow\left(\left(\phi_{1} \vee \phi_{2}\right) \rightarrow \psi\right)
$$

to CK. It is also known that ternary relation frames are determined by these same axioms, and that both of these result hold under the restricting to finite frames [4]. They are thus also determined by adding the same axioms to CCK. These axioms therefore also determine the representable frames.

A strong advantage of representable Ch-frames (and so ternary relation frames) over more general Chframes is that in applications it is sufficient to specify the transition $f$ at singleton sets in the right-hand argument. Many logics used for reasoning about particular classes of ternary frames have good prooftheoretic properties, see for example Pym [23], where for some standard conditional logics there are problems of 'explosion', see Priest [22].

\section{Analytic Tableau}

In this section we give a tableau proof procedure for conditional logic with recension connective. This is an extension of the elegant system given by Priest [22]; we follow his presentation closely, and we provide only the details required for the extension alongside minimal methodological reminders. In contrast to other sections we work in the absence of the RCEA rule, and with a relational notation so that transitions are now viewed as families of binary relations indexed by formulae. This allows for a simpler and more elegant tableau procedure, albeit for a less powerful logic. The RCEA rule is labelled (P) and discussed in 5.6.15 - 5.6.17 of Priest. 
Define a Pr-frame for the basic propositional language $\mathcal{L}=\mathcal{L}(\rightarrow, \triangleright)$ to be a structure $\mathcal{W}=\langle W, r\rangle$ where $W$ is a carrier set and $r: \mathcal{L} \rightarrow W \times W$ is a family of binary relations indexed by the formulae of the language. Note that these are the frames discussed informally at the beginning of Section 1. We write $r_{\phi}$ rather than $r(\phi)$ and mainly use infix notation for these relations. However, we also write $r_{\phi}(w)=\left\{w^{\prime} \in W \mid w r_{\phi} w^{\prime}\right\}$ and $r_{\phi}(U)=\bigcup_{w \in U} r_{\phi}(w)$ for $w \in W$ and $U \subseteq W$. A valuation is a map $v: \mathcal{L} \longrightarrow \mathcal{P}(W)$ and a model $\mathcal{M}$ consists of a Pr-frame and a valuation. An interpretation for the language is defined as in Section 4 except that we take

$$
\begin{aligned}
& \models_{w}^{(\mathcal{W}, v)} \phi \rightarrow \phi \quad \text { iff } \quad r_{\phi}(w) \subseteq \llbracket \psi \rrbracket^{(\mathcal{W}, v)} \\
& \models_{w}^{(\mathcal{W}, v)} \phi \triangleright \phi \quad \text { iff } \quad w \in r_{\phi}\left(\llbracket \psi \rrbracket^{(\mathcal{W}, v)}\right)
\end{aligned}
$$

where $\llbracket \psi \rrbracket^{(\mathcal{W}, v)}=\left\{w \in W \mid \models_{w}^{(\mathcal{W}, v)} \psi\right\}$. The notations $\Gamma \models_{w}^{\mathcal{M}} \phi$ and $\Gamma \models^{\mathcal{M}} \phi$ for sets of formulae $\Gamma$, formulae $\phi$, worlds $w$ and models $\mathcal{M}$, introduced in Section 4, adapt to this new interpretation of formulae in the evident way.

The system of tableau is similar to those often used for normal multi-modal logics. It uses one-sided tableau, written as trees, with nodes containing either pairs, $\phi, i$, where $\phi$ is a formula and $i$ is a formal world, or formal binary relation instances, $i r_{\psi} j$, between worlds $i$ and $j$, using formal relation symbols $r_{\psi}$ labelled by formulae $\psi$ of $\mathcal{L}$. It is sufficient to take the set of worlds to be the set of natural numbers (and we include zero).

The tableau rules are shown in Figure 2. As a matter of convention we often omit the notation for a vertical edge where the tree does not split. We allow the rules to be applied at any node of the tree below the parent node. Each rule is either negative if the outermost connective in the parent node is a negation (leftmost, when rewritten in Polish notation), or positive otherwise. The principal connective of a rule is the outermost connective of a positive rule, and the second-to-outermost connective in a negative rule. The rules for nodes whose principal connective is a classical propositional connective are the standard ones (in a normal modal context, with the world component of nodes carried through). The rules where the principal connective is the conditional, $\rightarrow$, are those given by Priest. The rules for $\triangleright$ are new; they have an obvious dual structure to the rules for $\rightarrow$. In each of these rules, as presented, the generation of new nodes is indicated by an edge that is drawn explictly and is read from top to bottom. For example, in the positive rule for $\rightarrow$ if one has a branch with both $\phi \rightarrow \psi, i$ and $i r_{\phi} j$, then this is extended to contain $\psi, j$; similarly, in the positive rule for $\triangleright$, if there is a branch ending with a node $\phi \triangleright \psi, j$, then this can be extended to a branch containing both $i r_{\psi} j$ and $\phi, i$. The world $j$ is taken to be fresh (not appearing already in the branch) in the negative rule for $\rightarrow$, and similarly the world $i$ is fresh in and the positive rule for $\triangleright$.

A branch of a tableau is closed if it contains any formula and its negation at the same world, otherwise it is open. A tableau is closed if every branch is closed, otherwise it is open. A literal is a propositional variable or the negation of a variable. A branch of a tableau to be complete if it is either closed, or every formula at a node that is not a literal has had the rule for its principal connective applied. A tableau is complete if every branch is complete. The logic $L$ generated by the tableau system consists of all formulae $\phi$ such that a root $\neg \phi, 0$ generates a closed tableau. This is extended to a relation $\Gamma \vdash_{L} \phi$ between sets of formulae $\Gamma$ and formulae $\phi$ as in Section 2. We omit the subscript on $\vdash_{L}$ for the remainder of this section. The example tableau in Figure 3 shows how $\vdash((\phi \rightarrow \psi) \triangleright \phi) \rightarrow \psi$ can be derived, via the presence of both $\psi, 0$ and $\neg \psi, 0$ on the same branch and using a fresh world 1.

Let $\mathcal{W}=\langle W, R\rangle$ be a Pr-frame. A realization of the set of formal worlds is a function $\rho: \mathbb{N} \longrightarrow W$. Let $\mathcal{M}=\langle\mathcal{W}, v\rangle$ be a model. Let $b$ be any branch of a tableau. A realization $\rho$ is said to be $\mathcal{M}$-faithful to $b$ if, for all $\phi, i, j$ :

(1) if $\phi, i$ lies on $b$, then $\models_{\rho(i)}^{\mathcal{M}} \phi$ (that is, $\phi$ is true at $\rho(i)$ )

(2) if $i r_{\phi} j$ on $b$, then $\rho(i) r_{\phi} \rho(j)$ on $\mathcal{W}$.

$\mathcal{M}$ is said to be faithful to $b$ if there is some $\mathcal{M}$-faithful realization.

Lemma 9.1 (Faithfulness Invariance under Tableau Rules). Let $b$ any branch of a tableau, $\mathcal{W}=\langle W, r\rangle$ be a Pr-frame, $\mathcal{M}=\langle\mathcal{W}, v\rangle$ be a model. If $\mathcal{M}$ is faithful to $b$, and a tableau rule is applied to it, then it produces at least one extension $b^{\prime}$ such that $\mathcal{M}$ is faithful to $b^{\prime}$. 


$$
\begin{gathered}
\neg \neg \phi, i \\
\text { । } \\
\phi, i
\end{gathered}
$$

$$
\begin{gathered}
\phi \wedge \psi, i \\
\mid \\
\phi, i \\
\psi, i
\end{gathered}
$$

$\phi \rightarrow \psi, i$
$i r_{\phi} j$
$।$
$\psi, j$

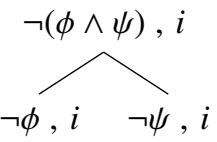
$\neg(\phi \rightarrow \psi), i$
I
$i r_{\phi} j$
$\neg \psi, j$

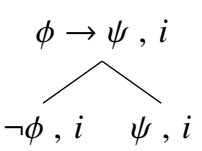

$$
\begin{gathered}
\neg(\phi \rightarrow \psi), i \\
\text { । } \\
\phi, i \\
\neg \psi, i
\end{gathered}
$$

$\neg(\phi \vee \psi), i$

।

$\neg \phi, i$

$\neg \psi, i$

$\phi, i \quad \psi, i$

$\neg(\phi \triangleright \psi), j$

$i r_{\psi} j$

।

$\neg \phi, i$

FIGURE 2. Tableau Rules

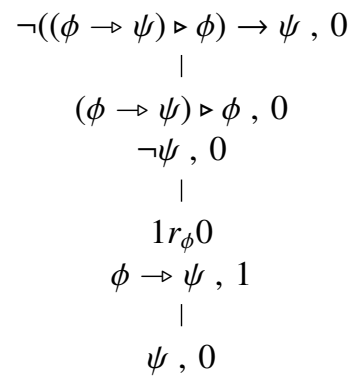

FIGURE 3. Tableau Example: Derivation of $\vdash((\phi \rightarrow \psi) \triangleright \phi) \rightarrow \psi$

Proof. The proof checks cases of the tableau rules that could have been used for the extension. We give only the cases for the two new rules as the rest go through in the standard way - see 1.11.2, 2.9.3 and 5.9.1 of Priest [22].

Suppose that the rule expanded is the positive one for $\triangleright$ in Figure 2. Thus we have $\phi \triangleright \psi, j$ on $b$; we additionally have $i r_{\psi} j$ and $\phi, i$ on $b^{\prime}$ for some new $i$. Since $\phi \triangleright \psi, j$ on $b$, by faithfulness $\phi \triangleright \psi$ is true at $\rho(j)$, where $\rho$ is some $\mathcal{M}$-faithful realization. By definition of $\triangleright$, there is some world $w$ such that $\phi$ is true at $w$ and $w r_{\psi} \rho(i)$. Define $\rho^{\prime}$ to be the realization that is identical to $\rho$, except at $i$, where $\rho^{\prime}(i)=w$. Since $i$ is fresh, the mapping $\rho^{\prime}$ is faithful to $b^{\prime}$ : in particular $\rho^{\prime}(i) r_{\psi} \rho^{\prime}(j)$ and $\phi$ is true at $\rho^{\prime}(i)$.

Suppose that the rule expanded is the negative one of for $\triangleright$ in Figure 2. We have $\neg(\phi \triangleright \psi), j$ and $i r_{\psi} j$ on $b$, and additionally $\neg \phi, i$ on $b^{\prime}$. By assumption, $\neg(\phi \triangleright \psi)$ is true at $\rho(j)$ for some faithful realization $\rho$ with $\rho(i) r_{\psi} \rho(j)$. It therefore cannot be the case that $\phi$ is true at $\rho(i)$, and so $\neg \phi$ is true at $\rho(i)$. Hence $\rho$ is faithful to $b^{\prime}$.

Theorem 9.2 (Soundness). If $\Gamma \vdash \phi$, then $\Gamma \vDash \phi$.

Proof. The proof is almost exactly as given by Priest [22] across 1.11.3, 2.9.4, 5.9.1, except that we allow infinite sets of antecedents and call upon the revised faithfulness invariance result above. Suppose $\Gamma \not \models \phi$. There is then a model $\mathcal{M}=\langle\langle W, r\rangle, v\rangle$ with a world $w$ such that every member of $\Gamma$ holds at $w$, but $\phi$ does not. Let $\Gamma^{\prime}$ be any finite subset of $\Gamma$, and let $\phi: \mathbb{N} \longrightarrow W$ be any function with $\rho(0)=w$. Let 
$\bigwedge \Gamma^{\prime}$ be the conjunction of elements in $\Gamma^{\prime}$. Consider a tableau beginning with root node $\neg\left(\left(\bigwedge \Gamma^{\prime}\right) \rightarrow \phi\right), 0$. Now $\left.\Gamma^{\prime}\right|_{w} ^{\mathcal{M}} \phi$, so $\models_{\rho(0)}^{\mathcal{M}} \neg\left(\left(\bigwedge \Gamma^{\prime}\right) \rightarrow \phi\right)$. The function $\rho$ is an $\mathcal{M}$-faithful realization at the root node. By Lemma 9.1 and induction, this extends to an $\mathcal{M}$-faithful realization, $\rho^{\prime}$, along the entirety of some branch of any tableau generated. This branch must be open: if it were closed, then by faithfulness we would have both $\models_{\rho^{\prime}(i)}^{\mathcal{M}} \psi$ and $\not_{\rho^{\prime}(i)}^{\mathcal{M}} \psi$ for some integer $i$ and formula $\psi$, which is impossible.

Any open branch $b$ of a tableau gives rise to induced models. The induced set of worlds is $W^{b}=\{i \in \mathbb{N} \mid$ $i$ occurs on $b\}$. The induced relation at $\psi$ is $r_{\psi}^{b} \subseteq W^{b} \times W^{b}$ given by the following rule:

- if, at a node of $b$, the formula $\psi$ occurs as the antecedent of a conditional connective, $\rightarrow$, or the antecedent of a negated conditional, or $\psi$ occurs as the right-hand argument of a recension, then for any $i, j \in W^{b}$, define $i r_{\psi}^{b} j$ iff $i r_{\psi} j$ on $b$; otherwise $r_{\psi}$ is empty.

A valuation $v: \operatorname{Var} \longrightarrow \mathcal{P}\left(W^{b}\right)$ is induced by $b$ if it is such that $i \in v(p)$ if $p, i$ on $b$, and $i \notin v(p)$ if $\neg p, i$ on $b$. There will be many induced valuations since only finitely many literals occur on $b$. Induced models bear the important relation to their parent open branches given in Lemma 9.3. In particular, this shows that an induced model with root $\neg \phi, 0$ provides a counter-model to $\phi$.

Lemma 9.3 (Interpretation Property in Induced Models.). Let b be any open complete branch of a tableau. Let $\mathcal{M}=\langle\mathcal{W}, v\rangle$ with $\mathcal{W}=\langle W, r\rangle$ be a model induced by the branch. Then:

- if $\phi, i$ is on $b$, then $\phi$ is true at $i$ in $\mathcal{M}$

- if $\neg \phi, i$ is on $b$, then $\phi$ is false at $i$ in $\mathcal{M}$.

Proof. The proof uses induction on the complexity of the formula $\phi$. Cases other than those for $\triangleright$ are exactly as in Priest [22] across 2.9.6 and 5.9.1.

Suppose that $\phi \triangleright \psi, i$ occurs on $b$. Since the branch is closed, the positive rule for $\triangleright$ must have been applied, so there is an $i$ with $i r_{\psi} j$ and $\phi, i$ on the branch. By the induction hypothesis, $\phi$ is true at $i$ in $\mathcal{M}$, and by the definition of the induced relation $i r_{\psi} j$ also in $\mathcal{M}$. Therefore $\phi \triangleright \psi$ is true at $j$ in $\mathcal{M}$.

Suppose that $\neg(\phi \triangleright \psi), j$ occurs on $b$. Suppose that $i r_{\psi} j$ in $\mathcal{M}$. Since the model is induced, we have $i r_{\psi} j$ on $b$. If $\phi, i$ lay on $b$, then by induction $\phi$ would be true at $i$ in $\mathcal{M}$. Since the branch is complete, the rule for negated $\triangleright$ must have been applied, and so $\neg \phi, i$ is on the branch. By the induction hypothesis, we would have $\phi$ not being true at $i$, giving a contradiction. Since this holds for all such $i$, the formula $\phi \triangleright \psi$ is not true at $j$ in $\mathcal{M}$.

Theorem 9.4 (Completeness). If $\Gamma \vDash \phi$, then $\Gamma \vdash \phi$.

Proof. Suppose $\Gamma \nvdash \phi$. For any finite $\Gamma^{\prime} \subseteq \Gamma$, any tableau for $\neg\left(\left(\bigwedge \Gamma^{\prime}\right) \rightarrow \phi\right), 0$ thus has an open branch, $b$. This branch must contain $\neg \phi, 0$ and $\psi, 0$ for all $\psi \in \Gamma$. Any induced model $\mathcal{M}$ from $b$ thus has $\Gamma^{\prime} \not \#_{0}^{\mathcal{M}} \phi$. Hence $\Gamma \not \neq \phi$.

The example tableau in Figure 4 shows how a countermodel for $\vdash(\phi \triangleright(\phi \rightarrow \psi)) \rightarrow \psi$ can be generated. The countermodel has the worlds, relations, and formulae holding at worlds as indicated on the branch.

$$
\begin{gathered}
\neg(\phi \triangleright(\phi \rightarrow \psi)) \rightarrow \psi, 0 \\
\mid \\
\phi \triangleright(\phi \rightarrow \psi), 0 \\
\neg \psi, 0 \\
\mid \\
1 r_{\phi \rightarrow \psi} 0 \\
\phi, 1
\end{gathered}
$$

FIGURE 4. Tableau Example: Countermodel for $\vdash(\phi \triangleright(\phi \rightarrow \psi)) \rightarrow \psi$ 


\section{FURTHER WORK}

Further applications of variants of CCK are possible. For example, in its use for handling hypothetical conditionals, the frame semantics is thought of as determining an update of the world along the hypothetical antecedent. A well-known approach to defeasible reasoning uses this semantic foundation, but interprets the relations defining frames instead to be update by a newly-known fact; the survey article by Koons [14] includes a rapid introduction. Extending this interpretation, the recension connective describes worlds that can arise under a particular update: the proposition $\phi \triangleright \psi$ is read as ' $\phi$ updated by $\psi$ '. For example, for $1 \leq n \leq 12$, suppose that $\phi(n)$ stands for the fact that some flight was scheduled to take off at $n$ o'clock, and that $\psi(n)$ stands for the fact that it actually took off at $n$ o' clock. Suppose that the flight was actually scheduled to take off at 1 o'clock, but that we subsequently learn that it took off at 2 o'clock. Initially, in our current world $w$ we have $\models_{w} \phi(1)$, omitting frame and valuation annotations, but once we learn of the delay we have $\models_{w^{\prime}} \phi(1) \triangleright \psi(2)$ in the updated world $w^{\prime}$. At the same time, we will have both $\models_{w^{\prime}} \neg(\phi(2) \triangleright \psi(2))$ and $\models_{w^{\prime}} \neg(\phi(1) \triangleright \psi(4))$. Fundamental in many applications of defeasible reasoning is non-monotonicity of consequence; we get this here with respect to conjunction in the form of recension: in CCK there are formulae $\phi, \psi, \theta$ so that $\phi \vdash \theta$, but not $\phi \triangleright \psi \vdash \theta$.

First-order conditional logic has been much studied in philosophical work [19, 22, 29]. No mathematical difficulties are foreseen in adapting the usual semantics, in particular using domains of individuals at worlds, but axiomatizations, proof-theory, and the advantage of any additional expressivity gained from adding recension to the first-order framework remain to be explored.

Generalizations of the logic could be investigated, for example weakening the boolean propositional base to an intuitionistic one to give a superintuitionistic logic with conditional and recension connectives. This would be interesting because CCK is a generalization of boolean substructural logics, including bunched logics, using a generalization of their semantics, and because intuitionistic substructural logics have important applications, particularly in theoretical computer science. A second direction for generalization would be combinations of CCK with other intensional logics with possible-world semantics. Of particular interest would be further development of combinations of conditional logics with logics of resource [23], in order to allow, for example, about reasoning about resource decomposition/sharing that could be done if a specific quantity more (or less) resource was present. The technique from the semantics of bunched logic of using Grothendieck Topological models may be more widely applicable to constructing models of conditional logic with 'impossible possible' worlds.

The formulation of the tableau system in this paper extends a well-known system for conditional logic. A version including that incorporates the semantical equivalent of the RCEA axiom remains to be developed. No analysis of the computational complexity of the tableau method for CCK has yet been done. The new tableau system bears a certain structural resemblance to tableau for bunched logics [8, 9, 17]. In light of the description of ternary relation semantics as the representable $\mathrm{Ch}$-frames, something like this is perhaps unsurprising. However, the tableau systems for bunched logics cited above either have an intuitionistic additive part, or use more concrete worlds (generated from words over an alphabet) in a more sophisticated analysis than has been carried out here. It remains to make a precise formal characterization of the relation between CCK and bunched logic tableau. Logical calculi for conditional logics with recension, other than the Hilbert and tableau systems above, remain to be investigated, in particular their proof theory. A starting point would be the methods used to treat Hilbert, Sequent, Natural Deduction and Display calculi used in earlier work [6], building on work by others, on a logic of layered graphs (LGL) involving non-commutative implication and conjunction connectives. Theorem-proving procedures and complexity results could also be investigated.

Unsurprisingly, CCK often has different properties to logics to which it is related. For example, CCK is decidable, as was shown above via the finite model property, but important boolean fragments of bunched logic are not, as has been demonstrated through encodings of machines in models and undecidable machine problems in the logical language [3]. More generally still, rather minimal boolean logics with binary modalities have been shown not to be decidable [15] using techniques from algebraic logic, arrow logic, and undecidability of word problems. Investigation of CCK from this more abstract viewpoint may prove fruitful, as it has for modal logics.

We have not discussed notions for transformation or comparison between models such as (bi)simulation morphism. For the relational frames used in this paper, preservation and reflection of satisfaction of the 
conditional connectives is achieved using the usual sort of back- and forth- conditions familiar from modal logic. A fuller analysis of correspondence theory for logics and frames could be done, along with studies of what relevant structure is transferred under simulation or under constructions.

A deeper look at relations between interpretations would study categories of structures, and translations and constructions between alternative semantics (Ch-frame, Scott-Montague minimal, algebraic) as functors. The construction of a canonical model from the algebraic semantics is an important example. Making this construction work smoothly from the perspective of category theory, that is, to determine a categorical duality between frames and algebras, has a number of nuances in the modal setting, both in the boolean and intuitionistic cases, and similar issues and solutions would be ripe for investigation here.

\section{ACKNOWLEDGEMENTS}

This work was supported by the UK Engineering and Physical Sciences Research Council under the research grants EP/K033042/1 and EP/P011829/1.

\section{REFERENCES}

[1] R.J. Aumann. Backward induction and common knowledge of rationality. Games Econ. Behav., 8(1):6-19, 1995.

[2] J.C. Beall, R. Brady, J.M. Dunn, A.P. Hazen, E. Mares, R.K. Meyer, G. Priest, G. Restall, D. Ripley, J. Slaney, and R. Sylvan. On the ternary relation and conditionality. Journal of Philosophical Logic, 41(3):595-612, 2012.

[3] J. Brotherston and M. Kanovich. Undecidability of propositional separation logic and its neighbours. J. ACM, 61(2):14:1-14:43, April 2014.

[4] B.F. Chellas. Basic conditional logic. Journal of Philosophical Logic, 4:133-153, 1975.

[5] B.F. Chellas. Modal logic: an introduction. Cambridge University Press, 1980.

[6] M. Collinson, K. McDonald, and D. Pym. A Substructural Logic for Layered Graphs. Journal of Logic and Computation, 24(4):953-988, 2014.

[7] M. Collinson and D. Pym. Algebra and logic for resource-based systems modelling. Mathematical Structures in Computer Science, 19:959-1027, 2009.

[8] D. Galmiche and D. Méry. Semantic labelled tableaux for propositional bi. Journal of Logic and Computation, 13(5):707-753, 2003.

[9] D. Galmiche, D. Méry, and D. Pym. The semantics of bi and resource tableaux. Mathematical Structures in Computer Science, 15(6):1033-1088, 2005.

[10] P. Gardenfors. Conditionals and changes of belief. Acta Philosophica Fennica, 30:381-404, 1978.

[11] J.Y. Halpern. Hypothetical knowledge and counterfactual reasoning. International Journal of Game Theory, 28(3):315-330, 1999.

[12] J.Y. Halpern. Substantive rationality and backward induction. Games and Economic Behavior, 37(2):425-435, 2001.

[13] B. Jónsson and C. Tsinakis. Relation algebras as residuated boolean algebras. Algebra universalis, 30(4):469-478, 1993.

[14] R. Koons. Defeasible reasoning. In Edward N. Zalta, editor, The Stanford Encyclopedia of Philosophy. Metaphysics Research Lab, Stanford University, winter 2017 edition, 2017.

[15] Á. Kurucz, I. Németi, I. Sain, and A. Simon. Decidable and undecidable logics with a binary modality. Journal of Logic, Language, and Information, 4:191-206, 1995.

[16] L. Lamport. Turing Lecture: The Computer Science of Concurrency: The Early Years. Communications of the ACM, 58(6), 2015.

[17] D. Larchey-Wendling and D. Galmiche. Exploring the Relation between Intuitionistic and Boolean BI: An Unexpected Embedding. Mathematical Structures in Computer Science, 19(03), 2009.

[18] E.J. Lemmon and D.S. Scott. The "Lemmon Notes": An Introduction to Modal Logic. Number 11 in American Philosophical Quarterly Monograph Series. Basil Blackwell, 1977.

[19] D. Lewis. Counterfactuals. Harvard University Press, 1973. Reissued London: Blackwell, 2001.

[20] P.L. Mott. On Chisholm's Paradox. Journal of Philosophical Logic, 2(2):197-211, 1973.

[21] A. Perea. Epistemic Game Theory. Cambridge University Press, 2012.

[22] G. Priest. An Introduction to Non-Classical Logic: From If to Is. Cambridge University Press, second edition, 2008.

[23] D.J. Pym. The Semantics and Proof Theory of the Logic of Bunched Implications, volume 26 of Applied Logic Series. Kluwer Academic Publishers, 2002.

[24] C. Saint Croix and R. Thomason. Chisholm's paradox and conditional oughts. Journal of Logic and Computation, 29(3):369386, 2019. Advanced Access version published online 12 February 2016 as article exw003.

[25] D. Samet. Hypothetical knowledge and games with perfect information. Games Econ. Behav., 17(2):230-251, 1996.

[26] K. Segerberg. Notes on conditional logic. Studia Logica, 48(2):157-168, 1989.

[27] R. Stalnaker. Knowledge, belief and counterfactual reasoning in games. Economics and Philosophy, 12:133-163, 1996.

[28] R.C. Stalnaker. A theory of conditionals. In N. Rescher, editor, Studies in Logical Theory (American Philosophical Quarterly supplementary monograph series), pages 98-112. Basil Blackwell, 1968.

[29] R.C. Stalnaker and R.H. Thomason. A semantic analysis of conditional logic. Theoria, 36:23-42, 1970.

[30] M. Ward and R.P. Dilworth. Residuated lattices. Trans. Amer. Math. Soc., 45(3):335-54, 1939. 
MatThew COLlinson, University of AbERDEEN

E-mail address: mat thew.collinson@abdn.ac.uk 